\title{
A METHODOLOGY TO INTEGRATE NUCLEAR MAGNETIC RESONANCE AND ACOUSTIC MEASUREMENTS FOR RESERVOIR CHARACTERIZATION
}

Semi-Annual Report

April 1, 2000-October 31, 2000

By

Jorge O. Parra
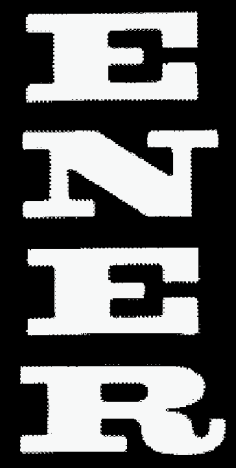

Work Performed Under Contract No. DE-AC26-99BC15203

Date Published: June 2002
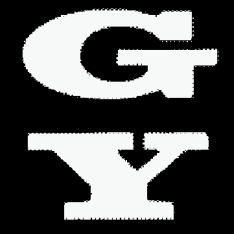

Southwest Research Institute

San Antonio, Texas

\section{National Energy Technology Laboratory National Petroleum Technology Office U.S. DEPARTMENT OF ENERGY Tulsa, Oklahoma}




\section{DISCLAIMER}

This report was prepared as an account of work sponsored by an agency of the United States Government. Neither the United States Government nor any agency thereof, nor any of their employees, makes any warranty, expressed or implied, or assumes any legal liability or responsibility for the accuracy, completeness, or usefulness of any information, apparatus, product, or process disclosed, or represents that its use would not infringe privately owned rights. Reference herein to any specific commercial product, process, or service by trade name, trademark, manufacturer, or otherwise does not necessarily constitute or imply its endorsement, recommendation, or favoring by the United States Government or any agency thereof. The views and opinions of authors expressed herein do not necessarily state or reflect those of the United States Government.

This report has been reproduced directly from the best available copy. 
DOE/BC/15203-4

Distribution Category UC-122

A Methodology to Integrate Nuclear Magnetic Resonance and Acoustic Measurements for Reservoir Characterization

\author{
By \\ Jorge O. Parra
}

June 2002

Work Performed Under DE-AC26-99BC15203

\author{
Prepared for \\ U.S. Department of Energy \\ Assistant Secretary for Fossil Energy \\ Purna Halder, Project Manager \\ National Petroleum Technology Office \\ P.O. Box 3628 \\ Tulsa, OK 74101
}

Prepared by

Southwest Research Institute

6220 Culbera Road

San Antonio, TX 78238 


\section{Table of Contents}

OBJECTIVES

ACCOMPLISHMENTS OF PHASE ॥

Task II.1 Measurements and Analysis of NMR at the Core and Borehole Scales.. 1

Task II.2 Catalog and Evaluate Core and Well Log Data from Select Reservoirs.. 2

Task II.3 Petrography from Cores and Petrophysics from Well Logs .................... 2

Task II.4 Validate Flow Mechanisms Using Porelastic Models and Applications .. 2

Task II.5 Modeling, Processing and Interpretation of Ultrasonic Data to Characterize Carbonate Rocks Containing Vuggy Porosity .................... 2

Task II.6 Technology Transfer Activities .............................................................. 2

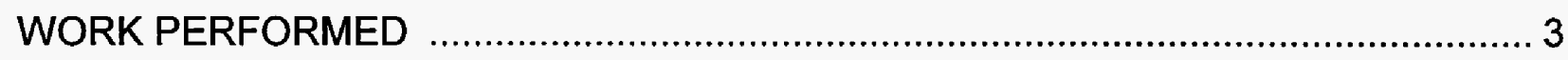

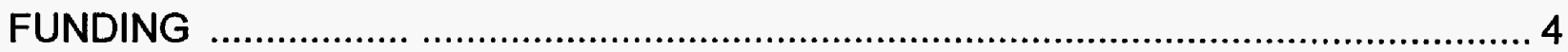

APPENDIX A "Integration of Acoustic and NMR Core Measurements: How to Relate Velocity with Porosity/Permeability in Vuggy Carbonates".........................A-1

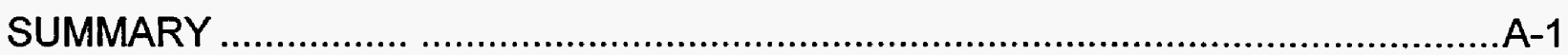

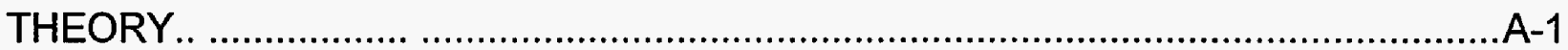

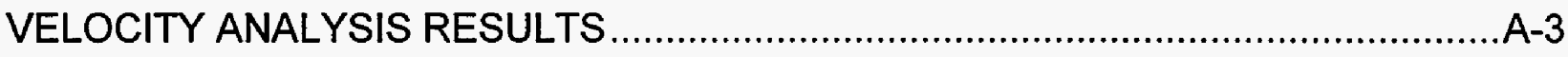

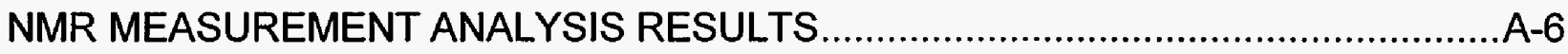

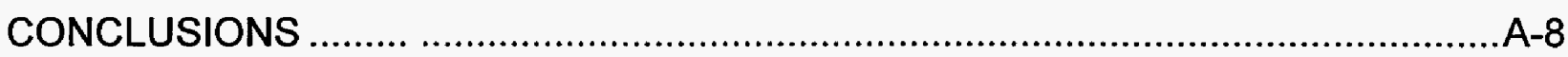

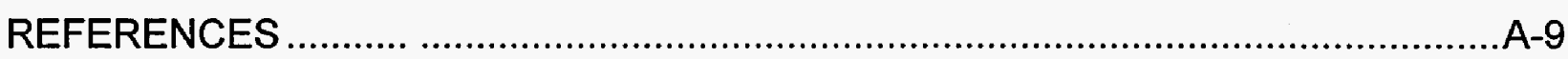

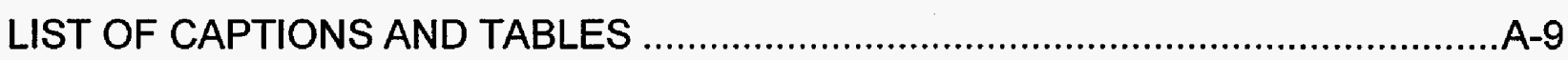

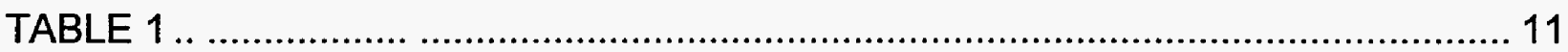

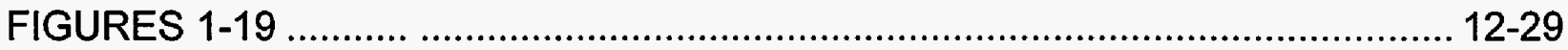




\section{SEMI ANNUAL REPORT}

\section{TITLE: A Methodology to Integrate Nuclear Magnetic Resonance and Acoustic Measurements for Reservoir Characterization}

DOE CONTRACT No: DE-AC26-99BC15203

CONTRACTOR: Southwest Research Institute

San Antonio, Texas

SWRI PROJECT MANAGER: ～Jorge O. Parra, Ph.D., Staff Scientist

DOE PROJECT MANAGER: Purna Halder

PROJECT DURATION: Three years

REPORTING PERIOD: $\quad$ April 1, 2000- October 31, 2000

\section{OBJECTIVES}

The objective of the project is to develop an advanced imaging method, including pore scale imaging, to integrate nuclear magnetic resonance (NMR) techniques and acoustic measurements to improve predictability of the pay zone in hydrocarbon reservoirs. We will accomplish this by extracting the fluid property parameters using NMR laboratory measurements and the elastic parameters of the rock matrix from acoustic measurements to create poroelastic models of different parts of the reservoir. Laboratory measurement techniques and core imaging will be linked with a balanced petrographical analysis of cores and theoretical modeling. These concepts will allow us to relate intrinsic acoustic properties with fluid flow interactions at the pore, core, and borehole scales, establishing the use of acoustic and NMR data to define flow units to eventually provide input to reservoir simulation modeling at interwell scales. The laboratory measurements will be compared with petrographic and imaging analysis results to determine the relative roles of petrographic elements such as porosity type (micro- and macroporosity), mineralogy, texture, and distribution of clay/cement in creating permeability heterogeneity.

\section{ACCOMPLISHMENTS OF PHASE II}

\section{Task II.1 Measurements and Analysis of NMR at the Core and Borehole Scales}

This task was modified to include analyses of NMR core measurements of nine samples as well as ultrasonic data recorded from 24 samples. A comprehensive description of this work is given in Appendix A, titled "Integration of Acoustic and NMR Core Measurements: How to Relate Velocity with Porosity/Permeability in Vuggy Carbonates." 


\section{Task II.2 Catalog and Evaluate Core and Well Log Data from Select Reservoirs}

Well logs from a carbonate aquifer in South Florida and the Ropes oil reservoir were cataloged. A report describing standard and modern well logs recorded in wells from these two reservoirs is given in Appendix B, titled "Florida and Ropes Data Well Logs and Processing."

\section{Task II.3 Petrography from Cores and Petrophysics from Well Logs}

Two full diameter cores were CT imaged to study their pore structure. We used Shell Oil Company facilities to process these two images. CT core images and processing data are given in Appendix C, "Processing of X-ray Computed Tomography (CT) Data." As a result of this analysis we concluded that thin section images from the two full diameter cores would be integrated with the CT scan images. For this purpose, we installed NIH software to perform image analysis to characterize pore space with sizes that can vary several order of magnitudes in a given sample. In addition, the CT data was used in Task 4 to model the cores ultrasonic responses.

\section{Task II.4 Validate Flow Mechanisms Using Poroelastic Models and Applications}

This is an important task that required information from Tasks 1, 2, 3, and 5. Some of the work done in Task II.1 addressed the squirt-flow mechanism that was predicted from the 24 samples. In Appendix A we predicted poroelastic responses based on measured rock physical properties. One important result was the separation of the P-wave velocity into flow units. In particular, the strong correlation between velocity and horizontal permeability was discussed, as was the sensitivity of ultrasonic data to the presence of vugs in carbonates. In Appendix A we also addressed separation of flow units at the borehole scale for predicting full waveform sonic at borehole and interwell scales. In addition, we calculated the effect of intrinsic and scattering attenuation associated with the South Florida reservoir flow units. These results are given in Appendix B. Appendix B also illustrates the monopole and dipole sonic full waveform data that we will use to calculate $\mathrm{Qp}$ and $\mathrm{Qs}$ at the borehole scale.

\section{Task II.5 Modeling, Processing and Interpretation of Ultrasonic Data to Characterize Carbonate Rocks Containing Vuggy Porosity}

This task presents the modeling and processing of ultrasonic data recorded in 24 cores. Modeling based on finite difference focuses on two full-diameter cores. A report is given in Appendix D, "Elastic Waves in Vuggy Porosisty."

\section{Task II.6 Technology Transfer Activities}

Two poster papers were presented at the 2000 Society of Exploration Geophysics Conference in Calgary:

1. Relating VSP with Well Logs in the Greater Green River Basin: Amplitude and Interval Velocity. 
2. A Model to Relate P-Wave Attenuation to Fluid Flow in Fractured Tight Gas Sands, Siliceous Shales, and Carbonate Reservoirs.

Two peer review papers were published:

1. Analysis of Multi-Scale Scattering and Poroelastic Attenuation in a Real Sedimentary Rock Sequence, published in the Journal of the Acoustical Society of America, 107, June 2000.

2. Characterization of Dispersion, Attenuation, and Anisotropy at the Buena Vista Hills Field, California, published on line in Geophysics at www.seg.org.

At the DOE review meeting, Dr. Parra had the opportunity to meet Dr. Kurt Nihei from Lawrence Berkeley Laboratories, who is working on the study of intrinsic and scattering effects based on ultrasonic core measurements in sandstones. Since we have the same goals, Dr. Nihei offered to perform ultrasonic measurement in vuggy carbonates. As a result, we have selected two long cores of 20 and 30 inches of lengths from well PF10. LBL is preparing these two cores for attenuation studies. The results will be useful for calibrating attenuation logs and interwell seismic data. Any new concepts and results will be shared with a Ph.D. student from the University of California at Berkeley, California.

After the preliminary results from the South Florida Data, we determined that crosswell seismic tomography is the most appropriate technique to map permeability in the interwell region. As a consequence South Florida Water Management will conduct a crosswell seismic survey between two wells in the reservoir region. We will plan and conduct the survey and we will process and integrate the data. This will give us the opportunity to verify the poroelastic models and to predict well log permeability and porosity profiles at the interwell scale.

\section{WORK TO BE PERFORMED}

1. Conduct NMR and capillary pressure core measurements for 10 more core samples.

2. Conduct mercury injection on selected samples.

3. Develop a better relaxitivity grouping using 18 samples.

4. Develop a program to calibrate NMR logs, calculate BVI logs, Sw, and permeability from well logs. The program will be general so it can use any type of permeability equation derived from core measurements.

5. Calibrate the NMR well log from the PF10 well.

6. Use thin section images from selected cores to predict matrix permeability.

7. Integrate thin section images with CT scan images from selected cores. 
8. Develop a program to predict $\mathrm{Qp}$ and $\mathrm{Qs}$ from full waveform sonic log data (both monopole and dipole).

9. Use the program developed in (8) to calculate Qp and Qs from full waveform data recorded in well PF10.

10. Correlate Qp and Qs with NMR-derived permeability/porosity at the borehole scale.

11. Correlate Vp and Vs with NMR-derived permeability/porosity at the borehole scale.

12. Plan crosswell seismic work to be conducted at the South Florida aquifer.

\section{FUNDING}

The total contract amount for the second year of this DOE project was $\$ 250,000$, and the balance is now $\$ 165,000$. Since we have the data to carry out Phase II of the project, we can proceed and use the project balance to accomplish most of the tasks of Phase II.

The total in-kind contribution from Springfield Exploration (SE) is $\$ 300,000$ for the three years. In addition, we have cost sharing from South Florida Water Management (SFWM). This organization has provided well log data (NMR and monopole/dipole) and core data (NMR, capillary pressure and ultrasonic). They will continue to give us support on NMR core measurement and mercury injection. These measurements are under way. In addition, SFWM will support the crosswell survey that will be conducted in January of 2001. 


\section{APPENDIX A \\ Integration of Acoustic and NMR Core Measurements: How to Relate Velocity with Porosity/Permeability in Vuggy Carbonates}

\section{SUMMARY}

We conducted ultrasonic measurements on 24 core samples and geotechnical measurements on more than 44 rock samples from the PF10 well in Hillsboro County (Palm Beach), South Florida. The well penetrates a carbonate aquifer from the Ocala Group, which is a Late Eocene limestone. We performed NMR measurements on nine core samples for the calibration of NMR well logs. The 44 samples were grouped on the basis of hydraulic units (HU) in the formation, and the HU zonation was based on the Klinkenberg permeability and helium porosity measured on the set of 44 samples. The HU concept is introduced here to explain the NMR relaxation data and to predict the petrophysical properties from well logs. The linkage between these two technologies is possible because HU zonation indicators referred to as flow zone indicators (FZI) and nuclear magnetic resonance (NMR) relaxation data are related to the surface phenomenon that controls the microscopic attributes of the rock. The phenomenological model can be represented by relationships between FZI and NMR relaxation data and parameters such as specific surface area, tortuosity, and relaxivity.

In this appendix we briefly review the existing concepts of FZIs and the basic equations of NMR to establish their link with acoustic parameters. In particular, we introduce new concepts such as how to relate FZI units with the Biot and squirt-flow mechanisms. This is done by separating the P-wave velocity in flow units, specifically as a function of horizontal permeability, and then predicting the velocity core measurements using a poroelastic model. We also present the NMR core measurement analyses and the steps to calibrate the NMR well log. Results of all analyses are presented and conclusions discussed.

\section{Flow Zone Indicators}

\section{THEORY}

Amaefule et al. (1993) developed an expression for FZI based on the Kozeny-Carman equation, which was first generalized to yield:

$$
\sqrt{(k /)}\left[1 /\left(S_{g v} \sqrt{F_{s}}\right)\right](/(1 \quad))
$$

Equation (1) is formed by parameters: $\mathrm{k}=$ permeability; $\phi=$ porosity, $\mathrm{F}_{\mathrm{s}}=$ shape factor, $\tau=$ tortuosity, and $\mathrm{S}_{\mathrm{gv}}=$ specific area per unit grain volume. The $\mathrm{F}_{\mathrm{s}}$ and $\tau$ represent pore throat tortuosity. In addition, Amaefule et al. (1993) introduced the concept of FZI units and defined the Reservoir Quality Index (RQI). These two indices are expressed as:

$$
\log (\mathrm{RQI})=\log (\mathrm{FZI})+\log \left(\phi_{\mathrm{z}}\right)
$$

where 


$$
R Q I=\sqrt{(k / \phi)}
$$

and

$$
F Z I=1 /\left(\tau S_{g v}\right) \sqrt{F_{s}}
$$

with

$$
\phi_{z}=\phi /(1-\phi)
$$

Equation (1) describes the relation between the pore space defined by the parameters $F_{s}, \tau$, and $\mathrm{S}_{\mathrm{gv}}$ and the FZI. This parameter can be defined as the relation between the volumetric proportion of pore space to its geometric distribution. Using Equation (1), Amaefule et al. (1993) showed that the porosity-permeability relation within each FZI interval can be uniquely defined.

The relations $\tau^{2}, F_{s}$, and $S_{\mathrm{gv}}^{2}$ to permeability and porosity remain constant for data points with similar pore-space attributes; therefore, when RQI is plotted versus normalized porosity in logarithmic space, data fall on a straight line with a $45^{\circ}$ slope. Where this straight line intersects the vertical axis $\left(\phi_{z}=1\right)$, the intercept is equal to log FZI. Samples with similar pore-space attributes exhibit similar vertical axis intercepts. Pore-space attributes control intrinsic flow properties, and samples with similar vertical intercepts belong to the same hydraulic unit. Any large deviation from this straight line indicates the existence of a separate hydraulic unit for which the relationship among $\tau, F_{s}, S_{g v}$, porosity, and permeability is different.

\section{Relation Between NMR Basic Equations and Flow Zone Indicators}

The fundamental equations that relate the apparent relaxation rate for a single pore in the porous media are given by

$$
1 / \mathrm{T} 2=1 / \mathrm{T} 2 \mathrm{~b}+\rho \mathrm{S} / \mathrm{V}
$$

where

$\mathrm{T} 2=$ observed transverse relaxation time.

$\mathrm{T} 2 \mathrm{~b}=$ relaxation time of bulk fluid

$\rho=$ surface relaxivity

$\mathrm{S}=$ surface area of the pore

$\mathrm{V}=$ volume of the pore body.

Since $\mathrm{T} 2 \mathrm{~b}$ is significantly greater than the relaxation time $\mathrm{T} 2$ in the porous medium, the above equation can reduced to:

$$
1 / \mathrm{T} 2=\rho(\mathrm{S} / \mathrm{V})
$$

Georgi and Menger (1994) introduced a relation between the surface area-to-volume ratio of the pore space with the porosity and the specific surface per grain volume ratio $\left(\mathrm{S}_{\mathrm{gy}}\right)$. This relation was also applied by Ohen et al. (1995) and is given by 


$$
\mathrm{S} / \mathrm{V}=\mathrm{S}_{\mathrm{gv}}(1-\phi) / \phi
$$

Thus, Equation (3) can be written as:

$$
\rho \mathrm{T} 2=\phi /\left[\mathrm{S}_{\mathrm{gv}}(1-\phi)\right]
$$

or

$$
\mathrm{T} 2 / \phi_{\mathrm{z}}=1 / \rho \mathrm{S}_{\mathrm{gv}}
$$

Thus, we can relate Equations (5), (6) and (7) with the RQI defined by Equation (2c):

$$
R Q I=\sqrt{(k / \phi)}=\rho T 2 /\left(\tau \sqrt{F_{s}}\right)
$$

This suggests that RQI is related to relaxation time in the same way it is related to porosity group $\left(\phi_{z}\right)$. Therefore, using the above equations we can write a logarithmic equation for T2 as

$$
\log \mathrm{T} 2=\log \left(\phi_{\mathrm{z}}\right)+\log \left[1 /\left(\rho \mathrm{S}_{\mathrm{gv}}\right)\right],
$$

This equation relates relaxation time to porosity and forms the basis for the relaxivity group concept, which is analogous to the HU concept. Equation (9) classifies samples that exhibit similar NMR relaxation (rock-fluid interaction) characteristics into a group. The average parameters for the group serve as calibration points for interpreting NMR logs. The factor $1 / \rho S_{v g}$, often referred to as relaxivity product, represents both the relaxation power and textural attributes of the formation. As implied from Equation (9), the $\log \log$ plot of T2 versus $\phi_{z}$ would result in a slope line, and $1 / \rho \mathrm{S}_{\mathrm{vg}}$ would be constant for all data points on that slope line.

Formation rock samples or intervals with similar NMR relaxation characteristics lend themselves to the same group, with their $\log \mathrm{T} 2$ versus $\log \phi_{\mathrm{z}}$ clustering around the intercepting slope line. We refer to the samples or intervals from a given formation or reservoir with similar NMR characteristics as belonging to the same relaxivity group.

\section{VELOCITY ANALYSIS RESULTS}

\section{Porosity-Permeability Relation}

To determine the FZI units we produced scatter plots of RQI versus normalized porosity $\phi_{z}$ in logarithmic space for the 44 samples using Equation (2a). The RQI parameters are defined by

$$
\begin{gathered}
R Q I=\sqrt{(k / \phi)} \\
\phi_{\mathrm{z}}=\phi /(1-\phi)
\end{gathered}
$$

As shown in Figure 1, the data fall on a straight line with a $45^{\circ}$ slope. From the RQI plots we determined the FZI values of each hydraulic unit. The plots show that five HU can be determined. These values are FZI $=8,4,2.5,1.25$, and 0.7 , and they are used to calculate 
permeability and porosity curves, which are shown overlaid on the horizontal versus porosity cross plots in Figure 2. Later, the FZI values were used to determine P-wave velocity versus log (permeability) curves to evaluate the Biot/squirt flow mechanism for waves traveling in vuggy carbonates.

\section{Velocity-Porosity Permeability Relation for the 44 Samples}

To correlate velocity and permeability parameters for the 44 samples, we first determined a relation between velocity and porosity based on the 24 samples available. This relation was obtained using a non-parametric transformation given in Datta-Gupta et al. (1997). Figure 3 shows the scatter plots between velocity and porosity and the function that best fits the points. This function has the following analytical representation,

$$
\mathrm{Vp}=-40.36 \varphi_{\mathrm{t}}^{2}+2094.4 \varphi_{\mathrm{t}}+1182.8
$$

where

$$
\varphi_{t}=0.00155 \varphi^{2}-0.1659 \varphi+3.344
$$

Based on this equation we calculated 44 velocity values versus the 44 porosity values recorded for each of the samples. The predicted velocities are cross plotted with the horizontal permeability values for the five flow units in Figure 4 . The plots show that velocity decreases as horizontal permeability increases, and when the flow units increase the horizontal permeability increases as well. The advantage of this analysis is that we can include all the 44 core samples available to predict velocity at the core scale. The disadvantage is that we are not including the observed velocity values. We will use this statistical approach for sonic log calibration and to calculate velocity-permeability relations at the borehole scale.

\section{Velocity-Porosity Relation for the 24 Velocity Samples}

The first objective of this analysis is to establish velocity-permeability relations that include the observed velocity data. The second objective is to model the observed velocity core data using poroelasticity to determine squirt-flow lengths. The best fit between observed and calculated velocity provides the squirt-flow parameters and a better bulk module for each sample.

Figure 5 shows the cross plots between velocity and horizontal permeability. The figure also depicts curves $\mathrm{a}, \mathrm{b}, \mathrm{c}, \mathrm{d}$, e, and $\mathrm{f}$ associated with six flow units. To determine the squirt-flow lengths for each sample based on the Biot squirt-flow mechanism, we used the theory given in Parra (2000). To calculate phase velocity, we used the rock physical and fluid properties of permeability, porosity, grain density, grain module, the drained Vp and Vs, fluid density, fluid velocity, and fluid viscosity. The model assumes that the poroelastic medium is homogeneous and isotropic. The drained Vp and Vs were calculated using Gasmman equations for fluid substitution. Thus, phase velocity data for the six flow units were calculated. As an example we illustrate in Figures 6a-b only phase velocities and attenuation based on cores from curve (b). In general, these curves show strong dispersion effects caused by the interactions between the rock matrix and fluid. The results of this analysis suggest a strong attenuation at ultrasonic signatures. 
We next selected the calculated velocities at $250 \mathrm{kHz}$, the frequency output used in the ultrasonic measurements. The calculated velocities are plotted in red, and the observed velocities are plotted in blue (see Figure 7). In the same scatter plots, the squirt-flow lengths for each of the calculated velocities are also included. There are a couple of velocities that we did not attempt to model. In this case we could not determine the undrained velocities using Gasmman equations. It is well know that Gasmman equations work better in homogeneous media and for low frequency velocity data. However, in our study, Gasmman equations worked well for predicting several of the dry velocities. For example, the observed and calculated velocities associated with the flow unit, FZI $=0.7$, agreed very well in the low permeability region. In the high permeability region there was a slight difference between observed and calculated velocities. This difference is due to degree of heterogeneity of the pore structure. We can say that any difference between the calculated and observed data provides an assessment of the amount of vuggy porosity in the core.

For example, the curve in Figure 7(a) shows an increase in squirt-flow length as permeability is increased. The large permeability correlates with large flow lengths. In the same curve, the departures of the calculated velocities from the observed velocities reveals the amount of vuggy porosity that may be present in the sample. In curve (b) the fit between observed and calculated velocities is perfect. In this case the velocity is controlled only by intrinsic effects caused by the fluid flow. In curve (c), the cluster of observed and calculated velocities between 1 and 10 Darcies suggests that scattering effects due to vuggy porosity cause the difference between observed and calculated velocities. In this curve, a squirt-flow length of $2.5 \mathrm{~cm}$ was predicted, which correlates to the high permeability values.

The final curve (f) shows a big difference of about $1000 \mathrm{ft} / \mathrm{s}$ between the observed and calculated velocity for core 41 . This core has several interconnected vugs, as shown in Figure 5, Appendix C. The image of this core gives some idea of what may happen as waves propagate in such a vuggy medium. Core 41 has two correlation lengths associated with the degree of heterogeneity of the vuggy porosity. In this heterogenous medium, Gasmman equations may not work very well. In fact, the dry Vp and Vs parameters were overestimated using Gasmman equations. As a result, we used measured dry velocity parameters for core 41 . Although we used measured data, the calculated velocity based on the dry velocity data shows a big departure from the observed velocity. This demonstrates that the highly heterogeneous conditions of core 41 can be predicted by comparing observed and calculated modeled velocities, which are based on a homogeneous poroelastic model. To further verify this concept, we calculated velocities for the entire cores 7 and 41, based on finite-difference modeling:

Uniform core (this is set as part of the model, not derived):

core $7: 4500 \mathrm{~m} / \mathrm{s}$

core 41: $3900 \mathrm{~m} / \mathrm{s}$

Water saturated, heterogeneous core:

core $7: 4520 \mathrm{~m} / \mathrm{s}$

core $41: 4360 \mathrm{~m} / \mathrm{s}$ 
Dry, heterogeneous core:

core 7: $4250 \mathrm{~m} / \mathrm{s}$

core $41: 3970 \mathrm{~m} / \mathrm{s}$

These velocities do not match well with the real core data because we do not yet have a good model to relate matrix density to P-wave velocity. However, we think the trends in velocity difference are reasonable. There is a $270 \mathrm{~m} / \mathrm{s}$ difference between wet and dry conditions in core 7 , and a $390 \mathrm{~m} / \mathrm{s}$ difference in core 41 . These are about the same differences that we observed using the poroelastic models.

In summary, the correlation between compressional wave velocity at the core scale has yielded a regression equation that can be used to relate velocity to porosity in a South Florida carbonate aquifer. In addition, the velocity versus horizontal permeability relation demonstrates that the compressional wave velocity strongly correlates with the horizontal permeability in each flow unit in the reservoir. This suggests that velocity tomography can be used to map permeability between water wells. Furthermore, the poroelastic modeling suggests that differences between observed and calculated data are due to the presence of vuggy porosity in the cores.

The next step in the analysis is to correlate log porosity with NMR-derived permeability using the RQI and FZI approaches. This will provide information as to how the flow units at the borehole scale relate with those at the core scale. It will also provide FZI values with which to cross plot the velocity from well logs with permeability for each flow unit. The velocity permeability relations will be compared with those derived from cores, and these results will provide new a understanding for deriving attenuation and dispersion mechanisms for carbonate rock with vuggy porosity. This in turn will be useful for modeling full waveform sonic and interwell seismic signatures based on velocity-permeability relations derived at the core and borehole scales.

\section{NMR MEASUREMENT ANALYSIS RESULTS}

In performing the NMR core measurements, the nine samples were wrapped in Teflon material to prevent grain loss. The rock samples (1 inch in diameter and 1-1.5 inches in length) were saturated with a two-percent $\mathrm{KCl}$ brine solution of density $1.016 \mathrm{~g} / \mathrm{cm}^{3}$. The next step was to place the samples in a CoreSpec-1000 instrument that operates at a proton Lamor frequency of one $\mathrm{Hz}$. The experiments measured the transverse relaxation time (T2), with an inter-echo spacing time of $500 \mathrm{~ms}$ and various trains to achieve a high NMR signal-to-noise ratio. Once a sample was unloaded, a saturated weight was again recorded to confirm no loss of weight.

The T2 spectrometry was performed on fully and partially saturated core plugs to obtain magnetization amplitude versus recovery time using the CoreSpec-1000. The output was processed using a multi-exponential fit based on the Levenberg-Marquardt algorithm. NMR porosity and relaxation time distributions were obtained from this process.

The nine samples were then loaded into a Beckman high-speed centrifuge and desaturated to $0.001 \mathrm{~cm}^{3}$ stabilization. It took approximately 36 hours to desaturate each sample, using speeds corresponding to 300 and 400 psig capillary pressure. Immediately after the 
samples were unloaded from the centrifuge, they were wrapped in plastic wrap. They were then weighed, unwrapped, and individually loaded in the CoreSpec-1000 for NMR T2 measurements. Each was weighed again immediately after the NMR measurement to check the material balance data.

Measurement results are given in Figures 8-16. Figures 8a -16a contain the T2 distribution converted to porosity, and Figures $8 \mathrm{~b}-16 \mathrm{~b}$ contain the $\mathrm{T} 2$ relaxation distributions for both desaturated and fully saturated samples overlaid on one plot. Optimum T2 relaxation cut-off times (T2c) for use in well log calibration were estimated by comparing the distribution curves.

Core analysis porosity was calculated from NMR measurements and from the Archimedes method. Figure 17 shows the relationship between NMR-derived porosity and helium porosity at a $0.50-\mathrm{ms}$ echo spacing. The figure shows a reasonably good match. Since different relaxation rates are observed in the data associated with the different rock groups in the well, different T2 cut-off times (T2c) are needed to obtain the appropriate bound-volume index (BVI). As a result, we obtained three relaxation groups from the nine samples (see Figure18). This was done by plotting $\log \mathrm{T} 2$ versus $\phi_{\mathrm{z}}$ and by fitting Equation (9). The cut-off values, permeability, and porosity, as well as several other important parameters, are given in Table 1.

The main objective of building a model based on FZI-NMR core data is to be able to construct a reliable algorithm for accurately calculating log permeability. By using FZI, which comes from hydraulic unit analysis, we can model all pore types encountered in a reservoir or aquifer formation. In this study, we also determined the FZI parameter in terms of the BVI data. For example, a model was developed to determine permeability in terms of the BVI and FZI parameters for each T2c cut-off. These cut-off transverse relaxation times, $T 2 \mathrm{c}$, for the nine samples range from 5 to $200 \mathrm{~ms}$. Thus, relaxation group 1 has an average T2c of $128 \mathrm{~ms}$, group 2 has an average T2c of $85 \mathrm{~ms}$, and group 3 has an average T2c of $6.5 \mathrm{~ms}$ (see Table 1). To take the three cut-offs into consideration, we constructed an expression for the FZI given by

$$
\mathrm{FZI}=[0.09464 \mathrm{C} /(1+1.0548 \mathrm{C})]^{\mathrm{x}},
$$

where $\mathrm{x}=0.33694$

and $\quad C=1-B V I / \varphi$.

To obtain permeability based on FZI values and NMR porosity, we use the relation given by

$$
k \quad 1014 F Z I^{2} \phi^{3} /(1 \phi)^{2} \text {. }
$$

The T2c cut-off parameters are given in Table 1. They were used to determine the BVI values, which are inserted in Equation (12a) to calculate the FZI units required to calculate permeability. Equation (12b) will be used to calculate permeability from the NMR well logs recorded in well PF10. The method that we will use to calibrate NMR well logs is as follows:

1. Cross plot the NMR T2 at 50 percentile (media) with normalized porosity $\left(\phi_{z}\right)$ from well log of well PF10. 
2. Use the logarithmic Equation 9 to plot straight lines with a 45-degree slop. The objective is to separate the $\mathrm{T} 2$ values into relaxation groups at the borehole scale. We expect that several groups will be derived.

3. Match the relaxation groups from well logs with those obtained from cores:

(a) If the $\mathrm{T} 2 / \phi_{\mathrm{z}}$ from well logs falls at or above 0.3725 seconds, the zone belongs to core relaxivity group 1 , and we use $\mathrm{T} 2 \mathrm{c}=128 \mathrm{~ms}$.

(b) If the $\mathrm{T} 2 / \phi_{z}$ from well logs falls between 0.3728 and 0.07288 seconds, the zone belongs to core relaxivity group 2 , and we use $\mathrm{T} 2 \mathrm{c}=85 \mathrm{~ms}$.

(c) If the T2/ $\phi_{z}$ falls between 0.07288 and 0.04946 seconds, the zone belongs to relaxative group 3 , and we use $\mathrm{T} 2 \mathrm{c}=6.5 \mathrm{~ms}$.

4. The T2c cut-offs will be used to determine the BVI from the well logs.

5. The last step is to calculate FZI and permeability using Equation 11.

\section{CONCLUSIONS}

We analyzed ultrasonic and low frequency NMR measurements from a South Florida carbonate aquifer for improving methods of integration that include acoustic and NMR data. We found that the carbonate contains micropores and macropores. Most of the cores contain interconnected and connected vugs of varying dimensions, as well as solid matrix porosity, which results in several pore sizes. This heterogeneous medium is sensed by the ultrasonic and NMR measurements. The ultrasonic velocities for two heterogeneous core samples were modeled to demonstrate that pore structure variability lowers the velocity about 10 percent. This effect was also observed when poroelastic models were compared with measured velocity data, suggesting that by comparing computed velocities of fluid-filled homogeneous porous media with observed velocities, we can predict the degree of heterogeneity associated with vuggy porosity. At the same time, we can relate this velocity increment to effective pore size distribution through CT image analysis. On the other hand, pore size distribution can be obtained from NMR core measurements. In these data the presence of vugs is associated with long T2 distributions that are more dispersed than those observed in most solid carbonate rock. The NMR core measurements produced nine different $\mathrm{T} 2$ cut-offs that were classified in three relaxivity groups. As a result of this analysis, the cut-offs were reduced to $128 \mathrm{~ms}, 85 \mathrm{~ms}$, and $6 \mathrm{~ms}$ for the calibration of the NMR well log recorded in well PF10. We suggested a method to calibrate NMR well logs based on the analysis.

The results illustrate that vuggy porosity can be characterized based on acoustic and NMR measurements at the core scale. However, to derive accurate matrix permeability relations for vuggy carbonates requires the analysis of $\mathrm{CT}$ and thin section images as well as synthetic $\mathrm{T} 2$ distributions based on pore size distributions that can be captured from image analysis. The CT image provides quantitative information and visualization of the pore structure of the core, and the CT image analysis determines whether the vugs are interconnected or disconnected in highly porous carbonates. Alternatively, thin section analysis can provide information on the solid matrix porosity, which will determine the matrix permeability of the carbonate rock in the core. As a result, pore size distributions of the rock matrix and the vugs can be determined. These results will be used to predict synthetic T2 distributions. These theoretical $\mathrm{T} 2$ distributions will 
be compared with low frequency NMR-measured T2 responses. Since we know the permeability from the cores, and we can independently calculate the matrix and the vuggy permeability, we can develop new processing techniques to accurately determine permeability from well logs.

\section{REFERENCES}

Amaefule, J.O., Attunbay, M.., Tia B.D., Kersey, D.G., Keelan, D.K., 1993, Enhanced reservoir description: using core and log data to identify hydraulic (flow) units and predict permeability in cored intervals/wells: SPE 26436, 1-16.

Xue, G., and Datta-Gupta, A., 1996, A new approach to seismic data integration during reservoir characterization using optimal non-parametric transformations: SPE 36500.

Ohen H.A., Ajufo, A., and Curby, F.M., 1995, A hydraulic (flow) unit based model for the determination of petrophysical properties from NMR relaxation measurements: SPE 0304626.

Georgi, D.T., Menger, S.K., 1994, Reservoir quality, porosity and permeability relationships. Paper presented at the Mintrop Seminar in Germany.

Parra, J.O., 2000, Poroelastic model to relate seismic wave attenuation and dispersion to anisotropy: Geophysics, 65, 201-202.

\section{LIST OF CAPTIONS AND TABLES}

Table 1. Relaxation groups for NMR log interpretation.

Figure 1. Crossplot of RQI versus $\log (\phi z)$ to determine FZI values.

Figure 2. Crossplot of horizontal permeability and porosity $(\phi)$. The solid lines are calculated permeability and porosity for different FZI parameters.

Figure 3. Crossplot of P-wave velocity versus porosity $(\phi)$. The solid line represents the best fit optimal transformation equation.

Figure 4. Crossplot of predicted P-wave velocity versus horizontal permeability. The $44 \mathrm{P}$-wave velocity values were produced using the velocity-porosity relationship determined in Figure 4.

Figure 5. Crossplot of the 24 measured P-wave velocity samples versus horizontal permeability.

Figure 6. Effect of frequency. (a) Phase velocity and (b) attenuation of 4 samples corresponding to the flow zone indicator FZI $=1.25$. The samples were calculated for squirt-flow lengths of 8 and $15 \mathrm{~mm}$.

Figure 8. NMR core measurements for sample 2 at depth of $1015.5 \mathrm{ft}$. (a) Porosity and T2 distributions for $100 \%$ saturated and desaturated sample, (b) comparison of $\mathrm{T} 2$ distributions for 
desaturated and $100 \%$ brine saturated sample for determination of $\mathrm{T} 2 \mathrm{c}$ cut off.

Figure 9. NMR core measurements for sample 4 at depth of $1018.3 \mathrm{ft}$. (a) Porosity and T2 distributions for $100 \%$ saturated and desaturated sample, (b) comparison of T2 distributions for desaturated and $100 \%$ brine saturated sample for determination of $\mathrm{T} 2 \mathrm{c}$ cut off.

Figure 10. NMR core measurements for sample 6 at depth of $10205.7 \mathrm{ft}$. (a) Porosity and T2 distributions for $100 \%$ saturated and desaturated sample, (b) comparison of $\mathrm{T} 2$ distributions for desaturated and $100 \%$ brine saturated sample for determination of T2c cut off.

Figure 11. NMR core measurements for sample 8 at depth of $1022.2 \mathrm{ft}$. (a) Porosity and T2 distributions for $100 \%$ saturated and desaturated sample, (b) comparison of $\mathrm{T} 2$ distributions for desaturated and $100 \%$ brine saturated sample for determination of T2c cut off.

Figure 12. NMR core measurements for sample 19 at depth of 1048. ft. (a) Porosity and T2 distributions for $100 \%$ saturated and desaturated sample, (b) comparison of $\mathrm{T} 2$ distributions for desaturated and $100 \%$ brine saturated sample for determination of $\mathrm{T} 2 \mathrm{c}$ cut off.

Figure 13. NMR core measurements for sample 21 at depth of $1050.4 \mathrm{ft}$. (a) Porosity and T2 distributions for $100 \%$ saturated and desaturated sample, (b) comparison of T2 distributions for desaturated and $100 \%$ brine saturated sample for determination of $\mathrm{T} 2 \mathrm{c}$ cut off.

Figure 14. NMR core measurements for sample 32 at depth of $1129.4 \mathrm{ft}$. (a) Porosity and T2 distributions for $100 \%$ saturated and desaturated sample, (b) comparison of $\mathrm{T} 2$ distributions for desaturated and $100 \%$ brine saturated sample for determination of T2c cut off.

Figure 15. NMR core measurements for sample 34 at depth of $1131.5 \mathrm{ft}$. (a) Porosity and T2 distributions for $100 \%$ saturated and desaturated sample, (b) comparison of $\mathrm{T} 2$ distributions for desaturated and $100 \%$ brine saturated sample for determination of T2c cut off.

Figure 16. NMR core measurements for sample 49 at depth of 1162 . ft. (a) Porosity and T2 distributions for $100 \%$ saturated and desaturated sample, (b) comparison of $\mathrm{T} 2$ distributions for desaturated and $100 \%$ brine saturated sample for determination of T2c cut off.

Figure 17. Cross plot of NMR-derived porosity and core analysis porosity.

Figure 18. T2 median versus normalized porosity $(\phi z)$. The lines illustrate three major relaxivity groups for NMR well log calibration.

Figure 19. Crossplot of FZI (NMR) verus BVI / $\phi$. The figure shows the models for FZI and permeability prediction from NMR BVI. The measured and calculated data are shown. 


\section{Relaxation Groups for NMR Log Interpretation}

\begin{tabular}{|c|c|c|c|c|c|c|c|c|c|c|c|}
\hline \multirow{2}{*}{$\begin{array}{c}\text { Relaxivit } \\
\text { y } \\
\text { Group }\end{array}$} & \multirow{2}{*}{$\begin{array}{c}\text { DEPTH } \\
\text { Feet } \\
\end{array}$} & \multirow{2}{*}{$\begin{array}{c}\text { Sample } \\
\text { I.D. }\end{array}$} & \multicolumn{2}{|c|}{ NOB (800 psi) } & \multicolumn{2}{|c|}{ Models } & \multirow{2}{*}{$\begin{array}{c}\mathbf{S}_{\text {wirr }} \\
\text { NMR } \\
\% \\
\end{array}$} & \multirow{2}{*}{\begin{tabular}{|c|} 
Swirr \\
(material balance) \\
$\%$ \\
\end{tabular}} & \multirow{2}{*}{$\begin{array}{c}\text { NMR } \\
\\
\phi \\
\% \\
\end{array}$} & \multirow{2}{*}{$\begin{array}{c}\text { FZl core } \\
\mu \mathrm{m} \\
\end{array}$} & \multirow{2}{*}{$\begin{array}{c}\text { FZlnmr } \\
\mu \mathrm{m}\end{array}$} \\
\hline & & & $\begin{array}{c}\text { Klinkenberg } \\
\text { mD }\end{array}$ & $\begin{array}{l}\phi \mathrm{He} \\
\% \\
\end{array}$ & $\begin{array}{l}\mathrm{K}_{\mathrm{NMR}} \\
\mathrm{mD} \\
\end{array}$ & $\begin{array}{l}\text { Kcoates' } \\
\text { mD }\end{array}$ & & & & & \\
\hline 1 & 1018.30 & 4 & 158.2 & 13.2 & 1.14 & 19.4 & 35.8 & 31.7 & 13.7 & 7.14744 & 0.569808 \\
\hline 1 & 1020.70 & 6 & 0.02 & 12.1 & 0.17 & 0.26 & 66.1 & 65.1 & 10.0 & 0.092738 & 0.364073 \\
\hline 1 & 1022.20 & 8 & 363.8 & 28.6 & 19.5 & 729.4 & 19.6 & 18.6 & 25.7 & 2.795788 & 0.791072 \\
\hline 1 & 1129.40 & 32 & 220.1 & 42.1 & 345.3 & 28372.2 & 9.6 & 5.7 & 42.2 & 0.987404 & 1.230382 \\
\hline 1 & 1131.50 & 34 & 436.3 & 44.8 & 491.9 & 41840.4 & 8.6 & 3.0 & 43.9 & 1.207422 & 1.343349 \\
\hline 2 & 1162.00 & 49 & 13.2 & 40.4 & 98.6 & 2605.1 & 24.9 & 30.0 & 41.1 & 0.265084 & 0.697192 \\
\hline 2 & 1015.50 & 2 & 0.02 & 7.1 & 0.04 & 0.02 & 79.0 & 79.2 & 7.1 & 0.218058 & 0.290821 \\
\hline 3 & 1048.00 & 19 & 13.0 & 20.3 & 4.1 & 42.2 & 39.8 & 30.7 & 20.7 & 0.986921 & 0.534884 \\
\hline 3 & 1050.40 & 21 & 31.5 & 31.9 & 45.4 & 749.6 & 23.3 & 17.9 & 33.6 & 0.66611 & 0.721365 \\
\hline
\end{tabular}

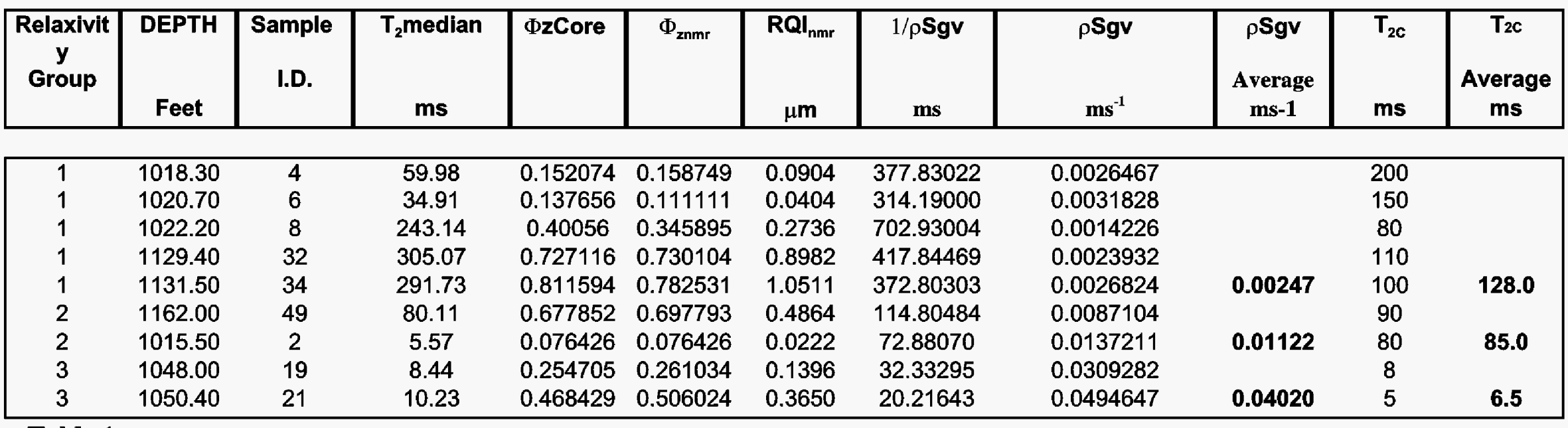

Table 1 


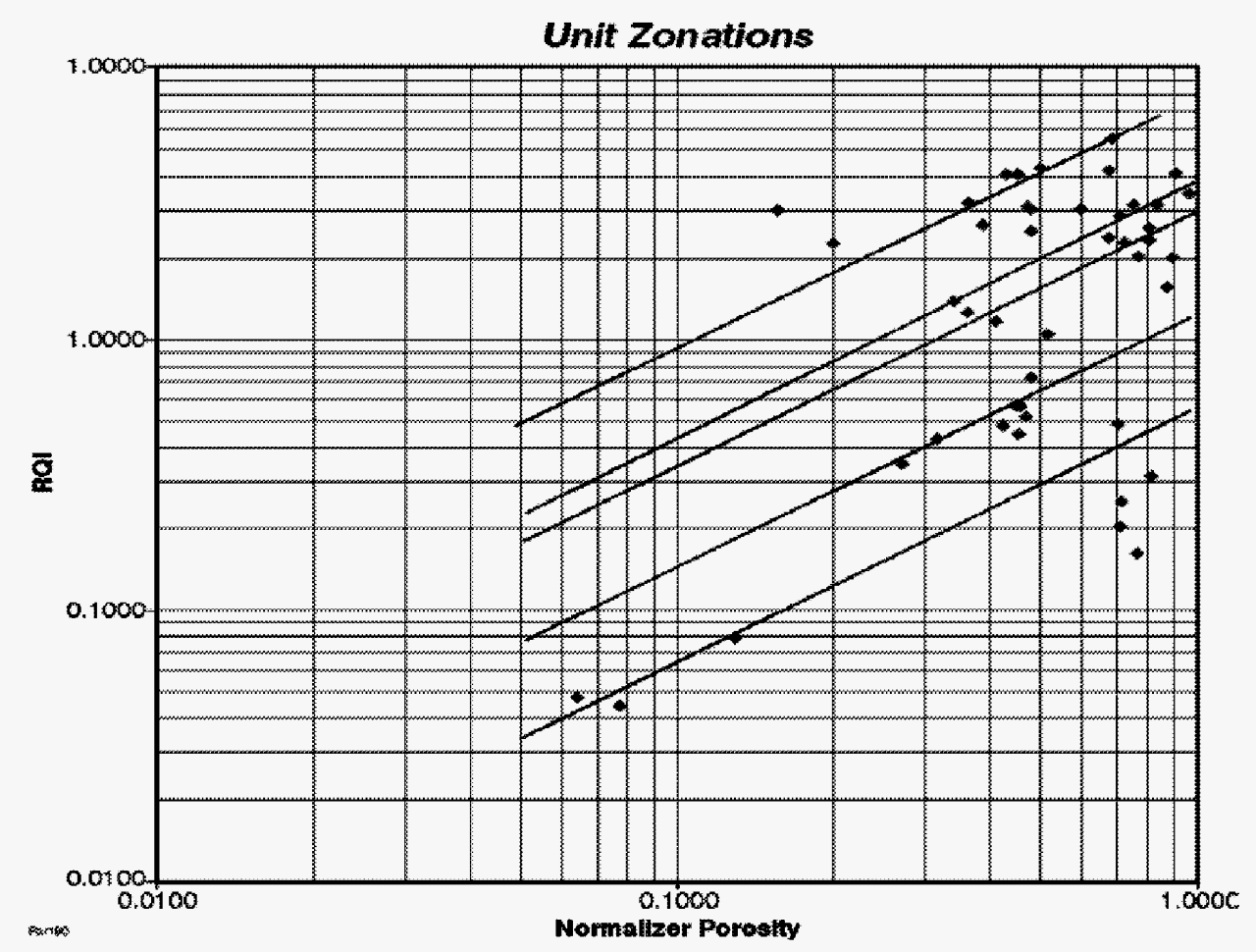

Figure 1 


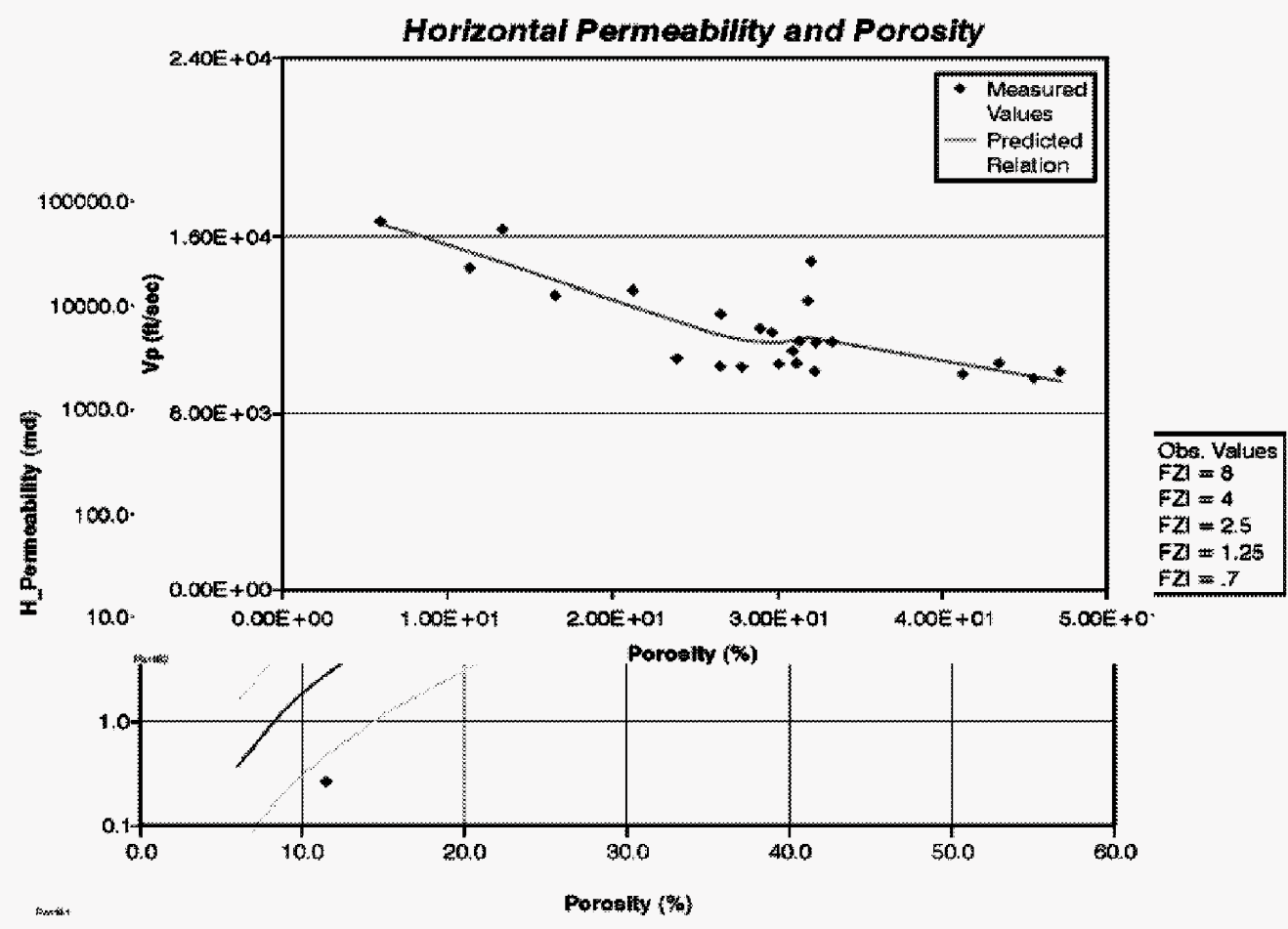

Figure 2 
Figure 3

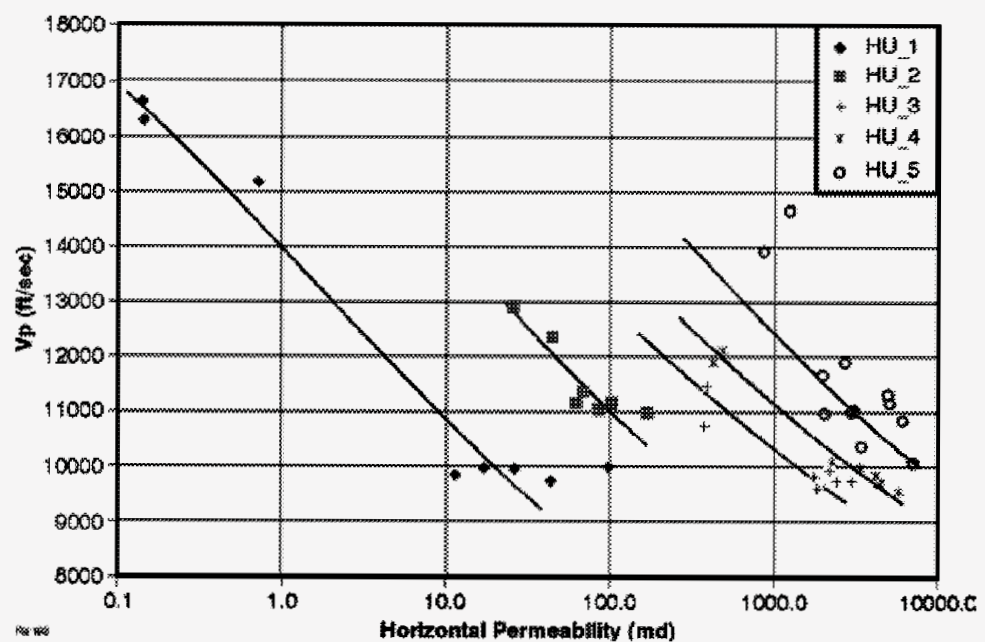




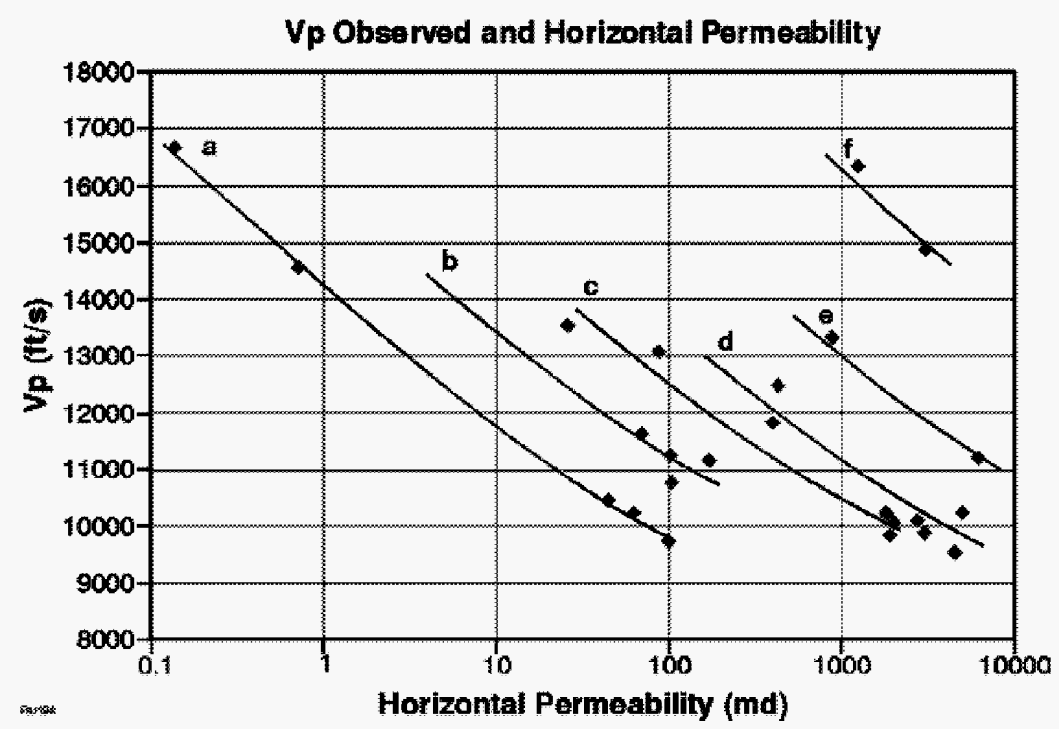

Figure 4

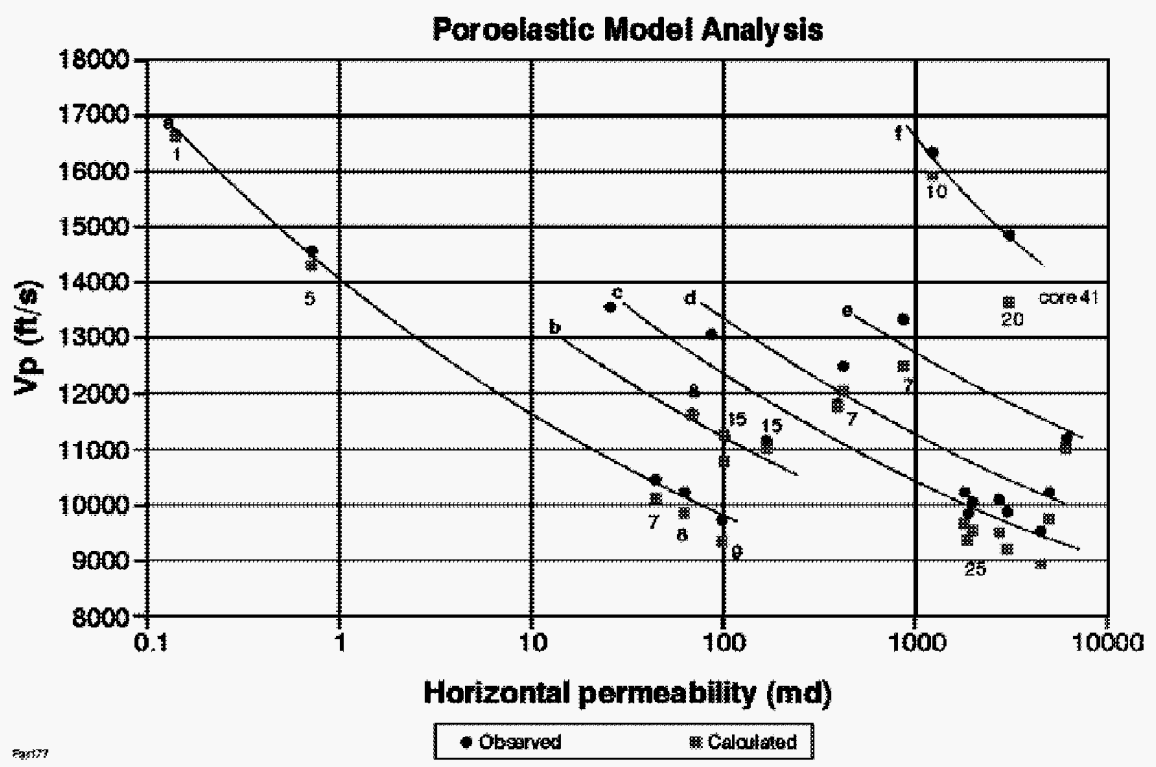

Figure 5 

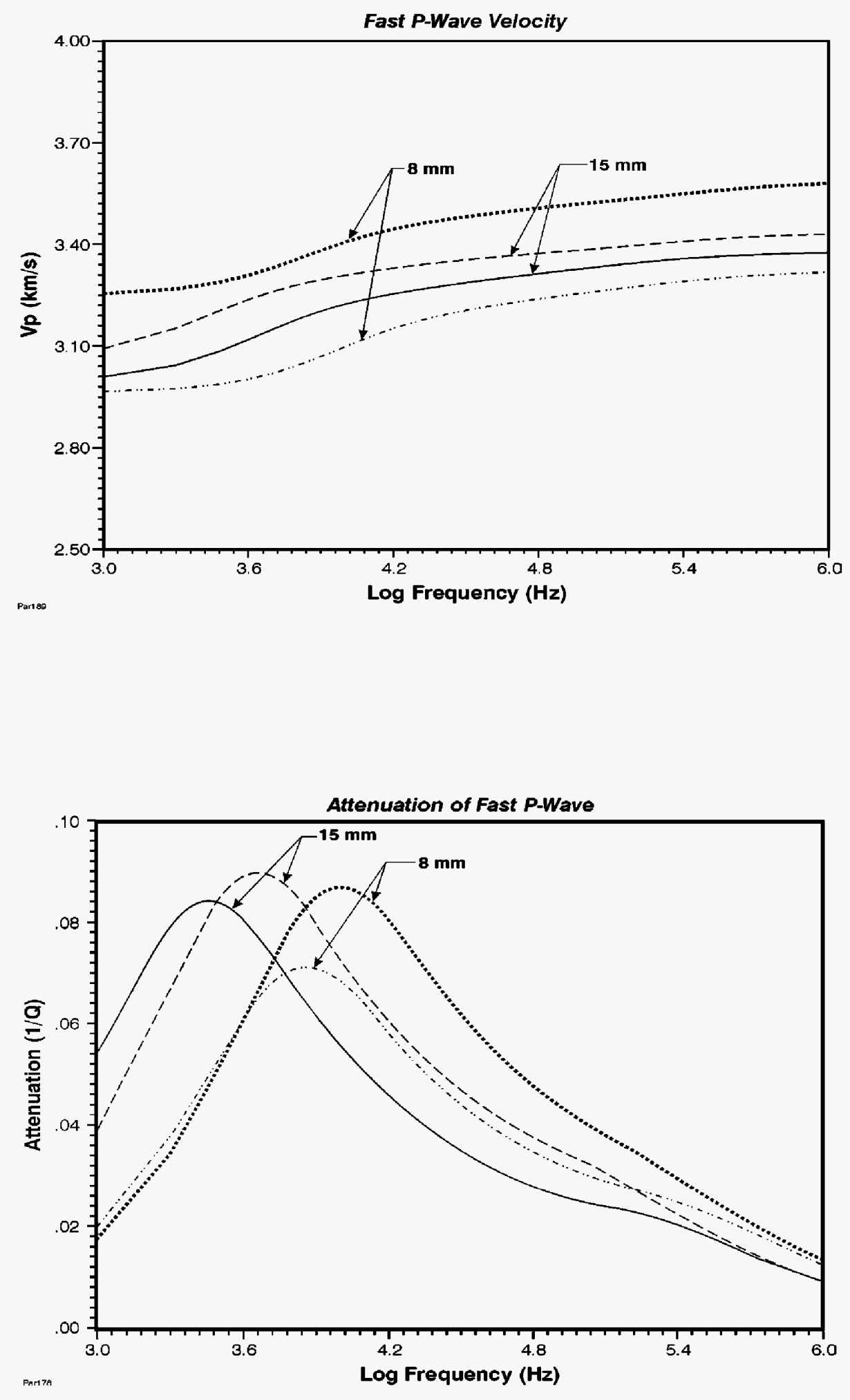

Figure 6a 

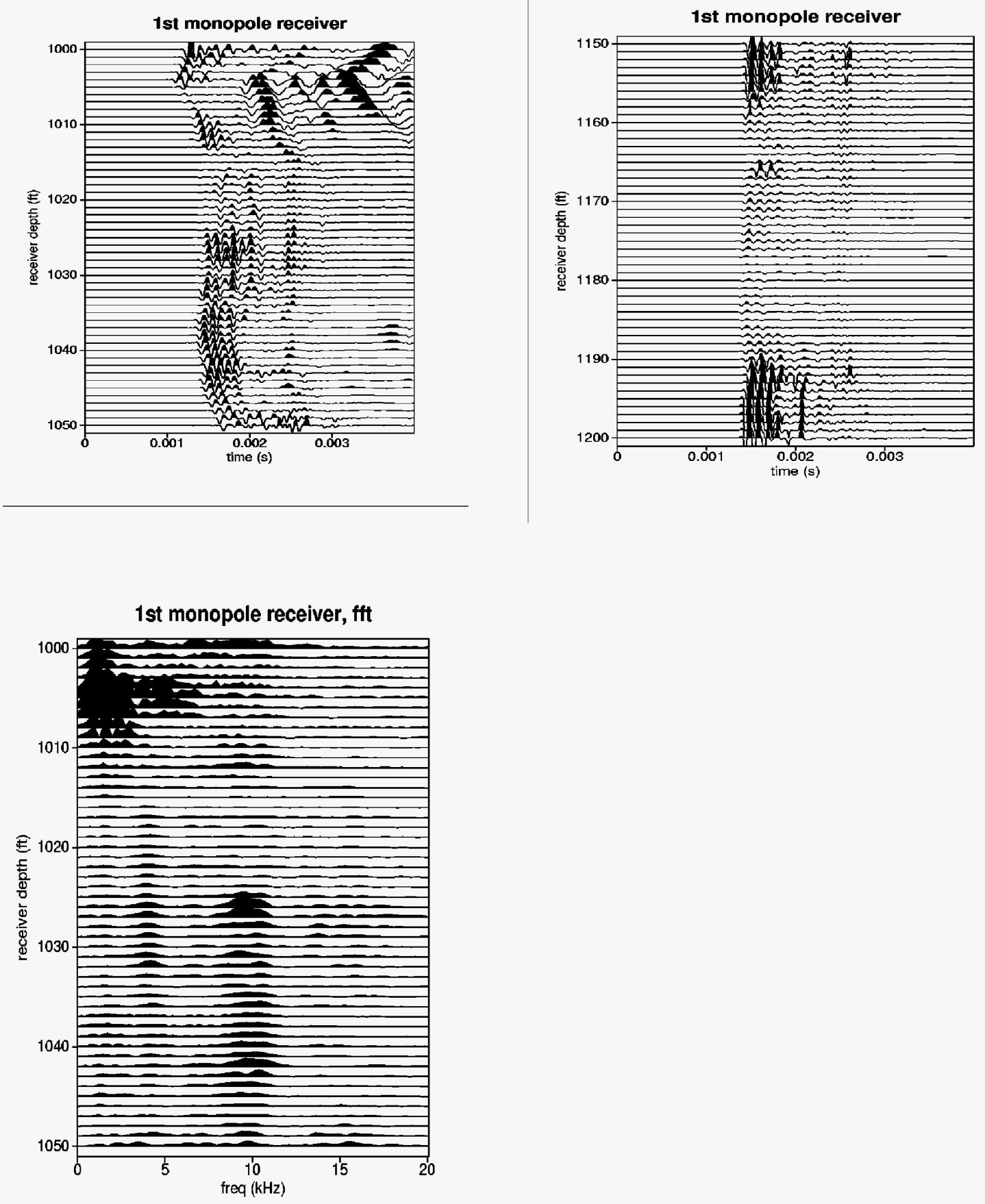

\section{Figure 6b}




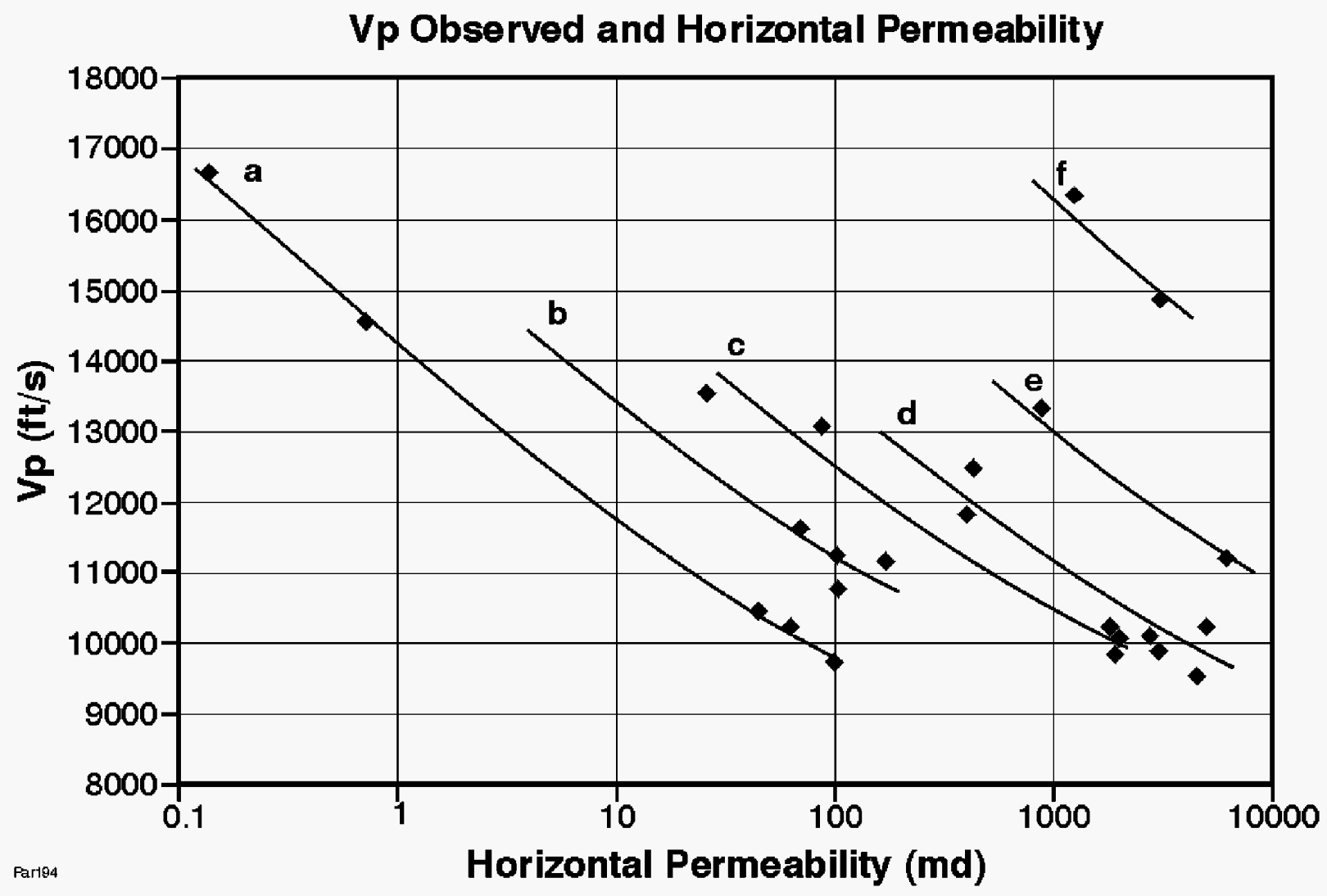

Figure 7 
Sample 2 Depth $1015.50 \mathrm{ft}$.

承
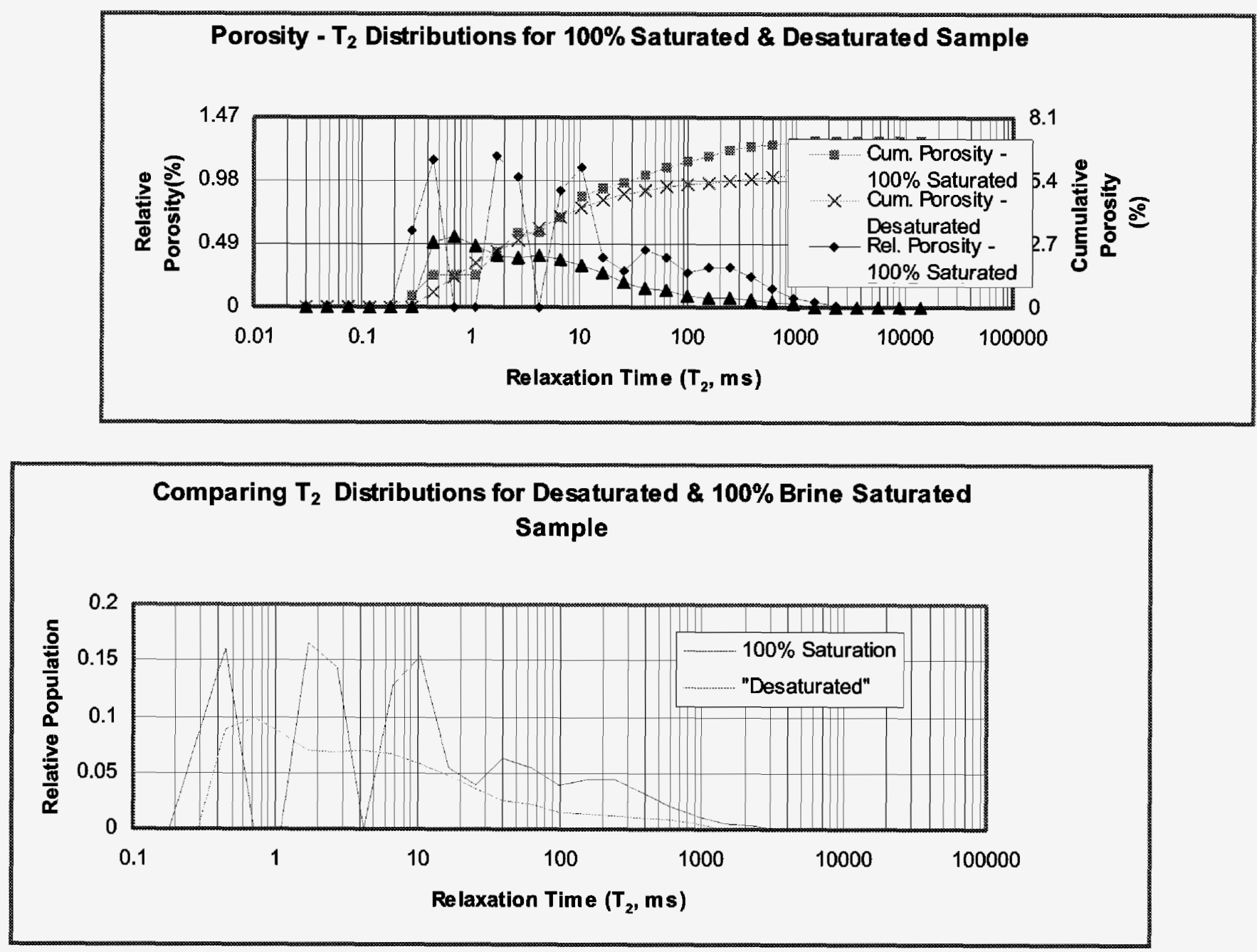
Sample 4 Depth $1018.30 \mathrm{ft}$.

褐

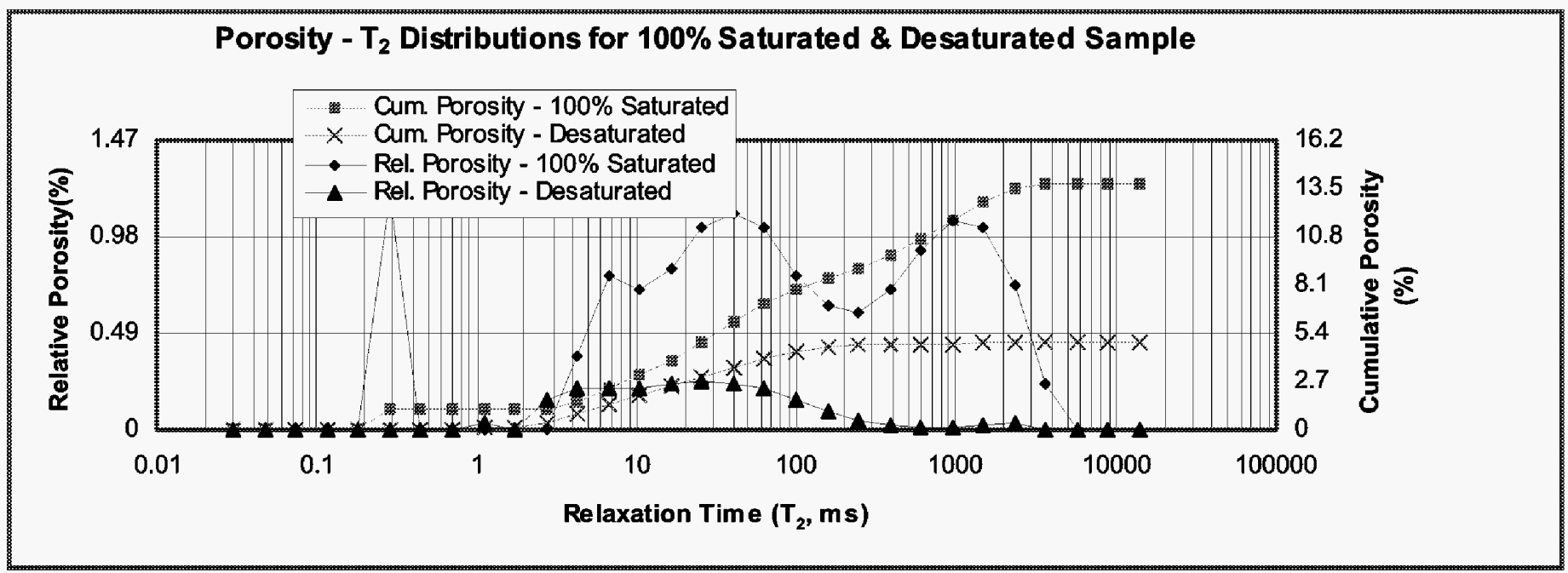

天

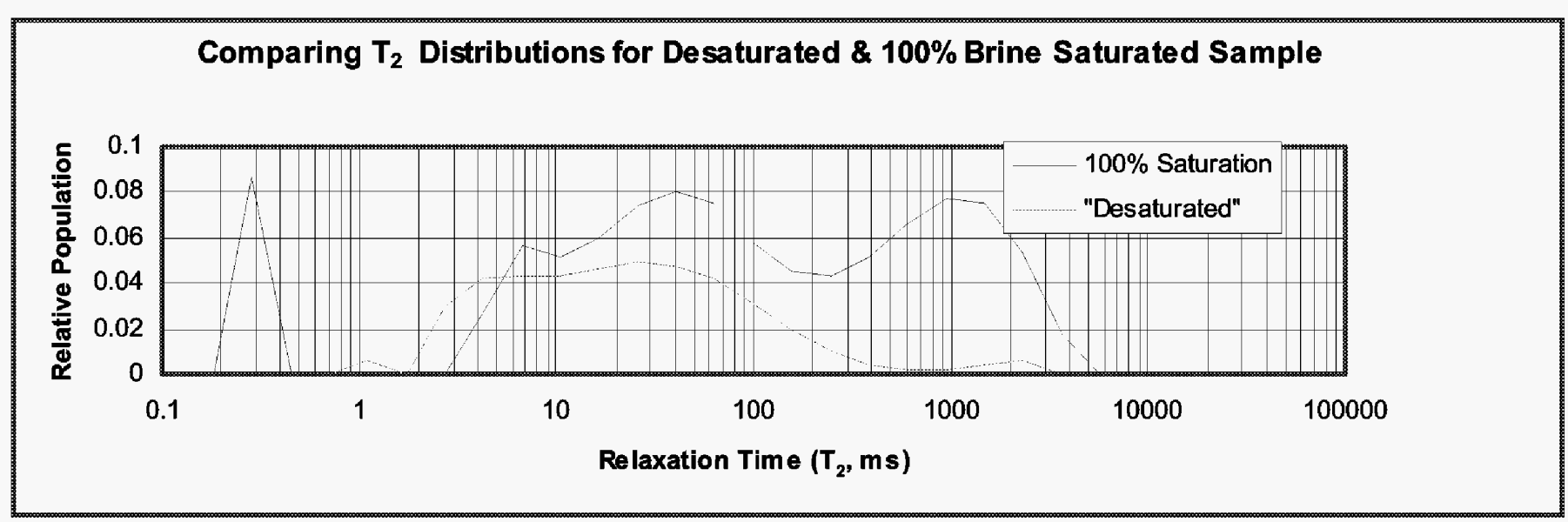


Sample 6 Depth $1020.70 \mathrm{ft}$.
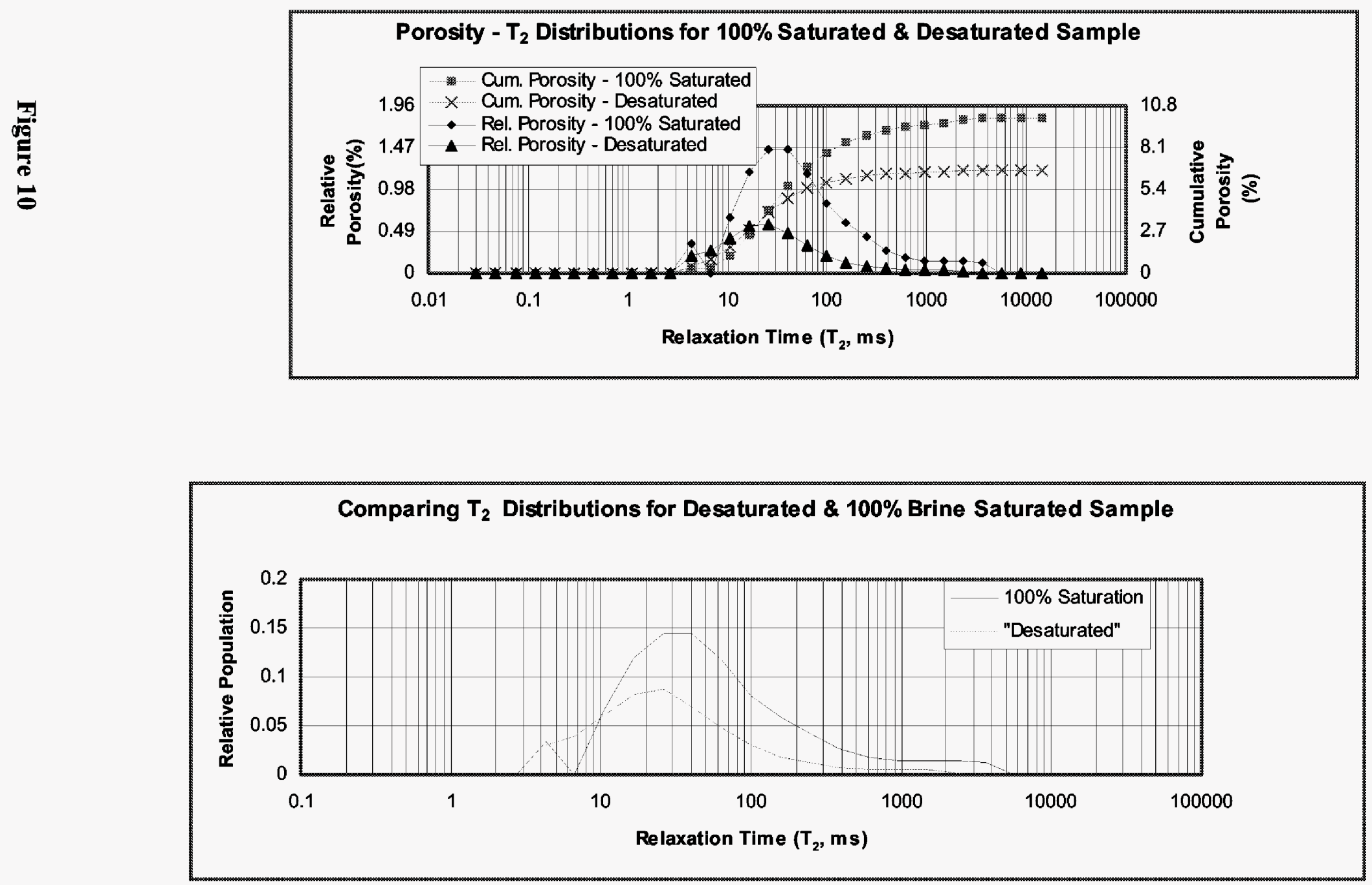
Sample 8 Depth $1022.20 \mathrm{ft}$.

葛
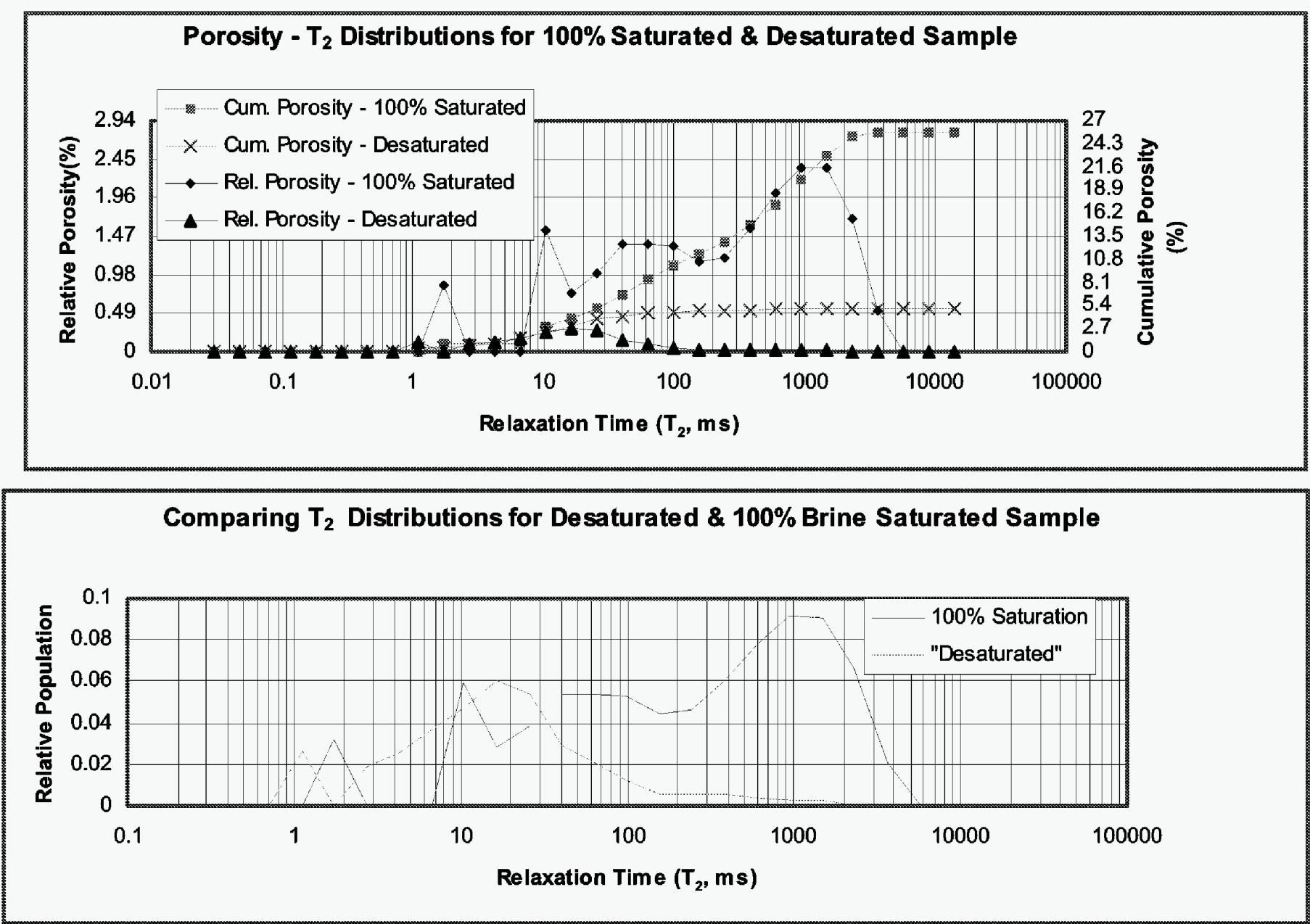
Sample 19 Depth $1048.00 \mathrm{ft}$.

葛
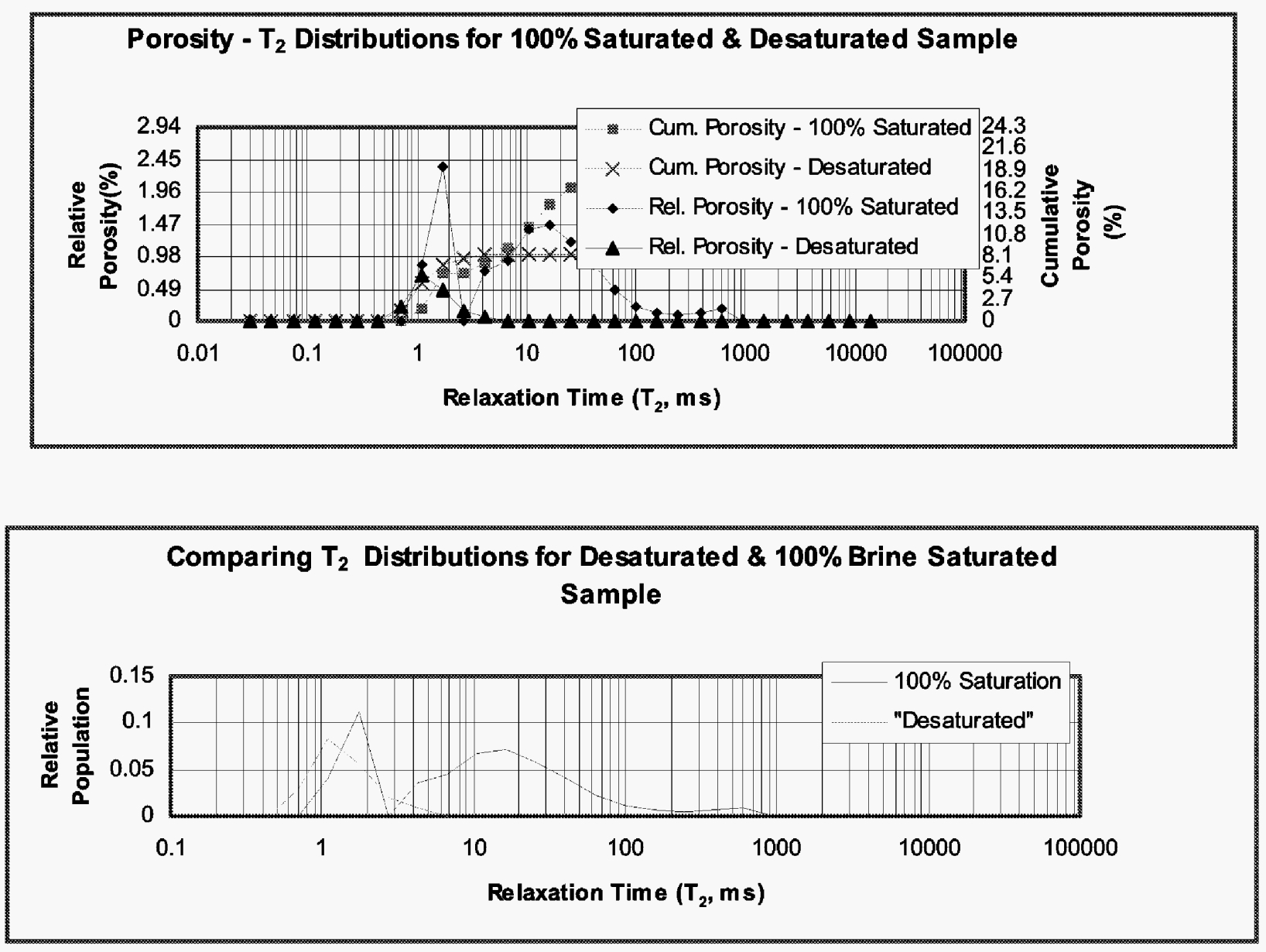
Sample 21 Depth $1050.40 \mathrm{ft}$.
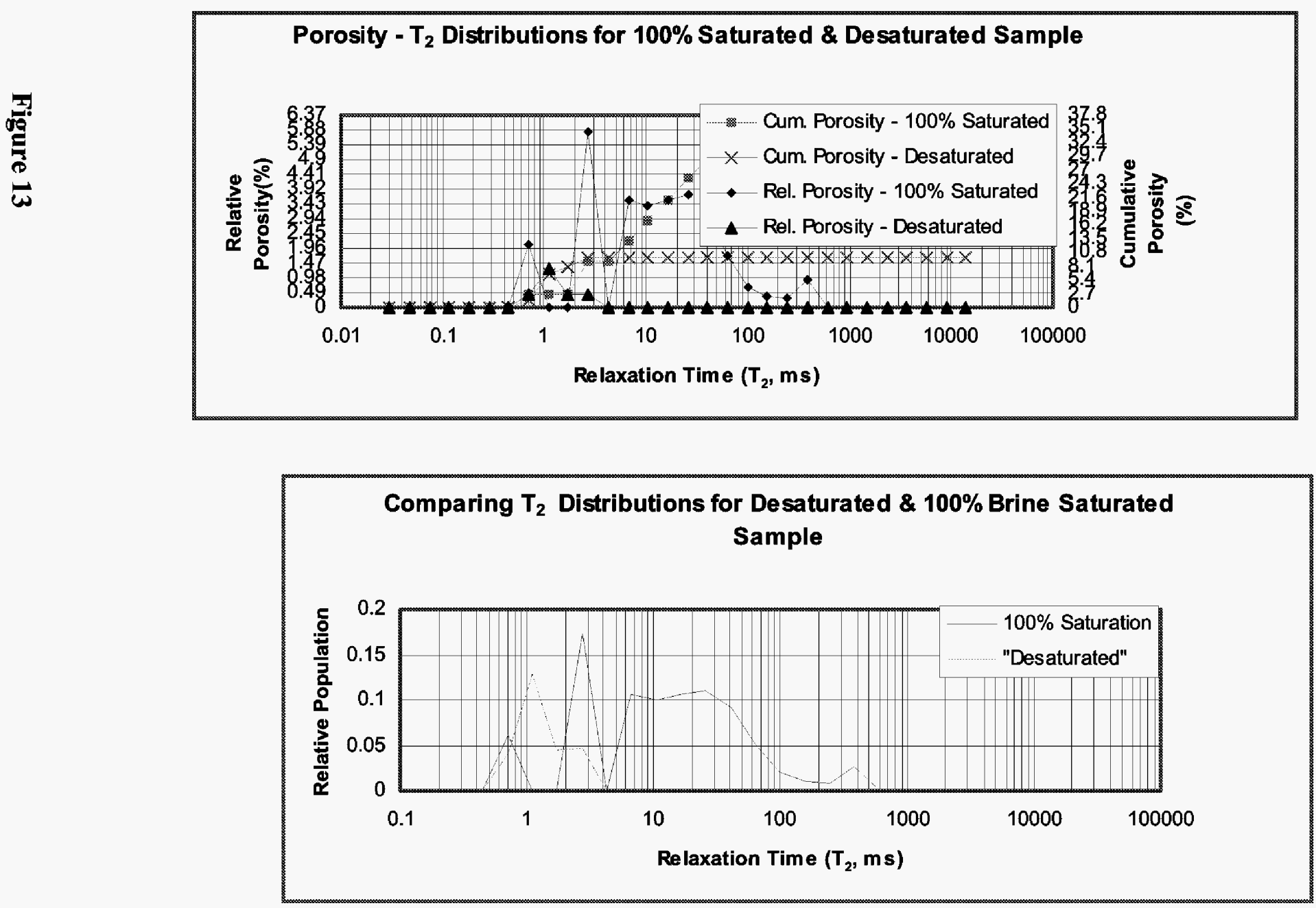


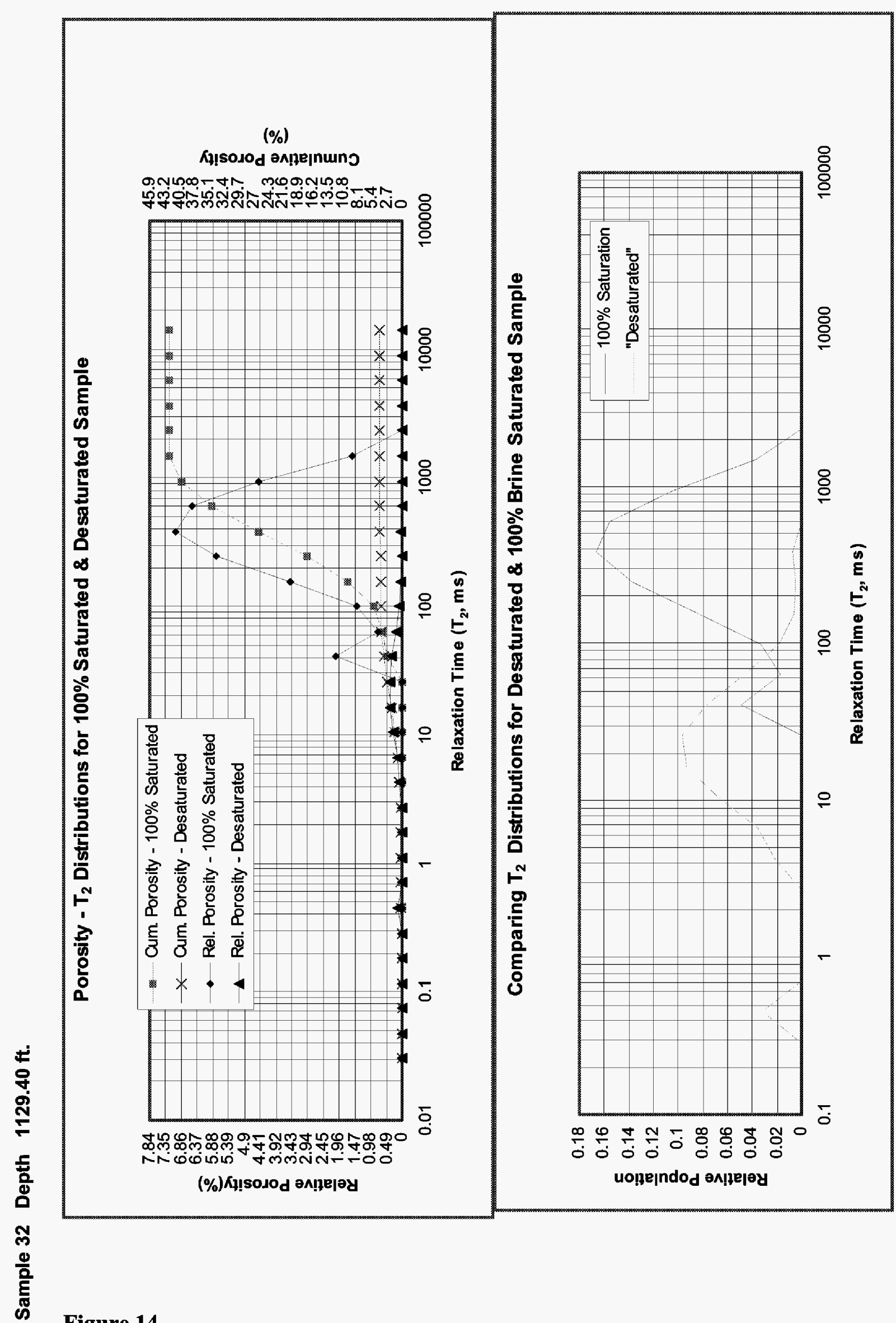

Figure 14 
Sample 34 Depth $1131.50 \mathrm{ft}$.
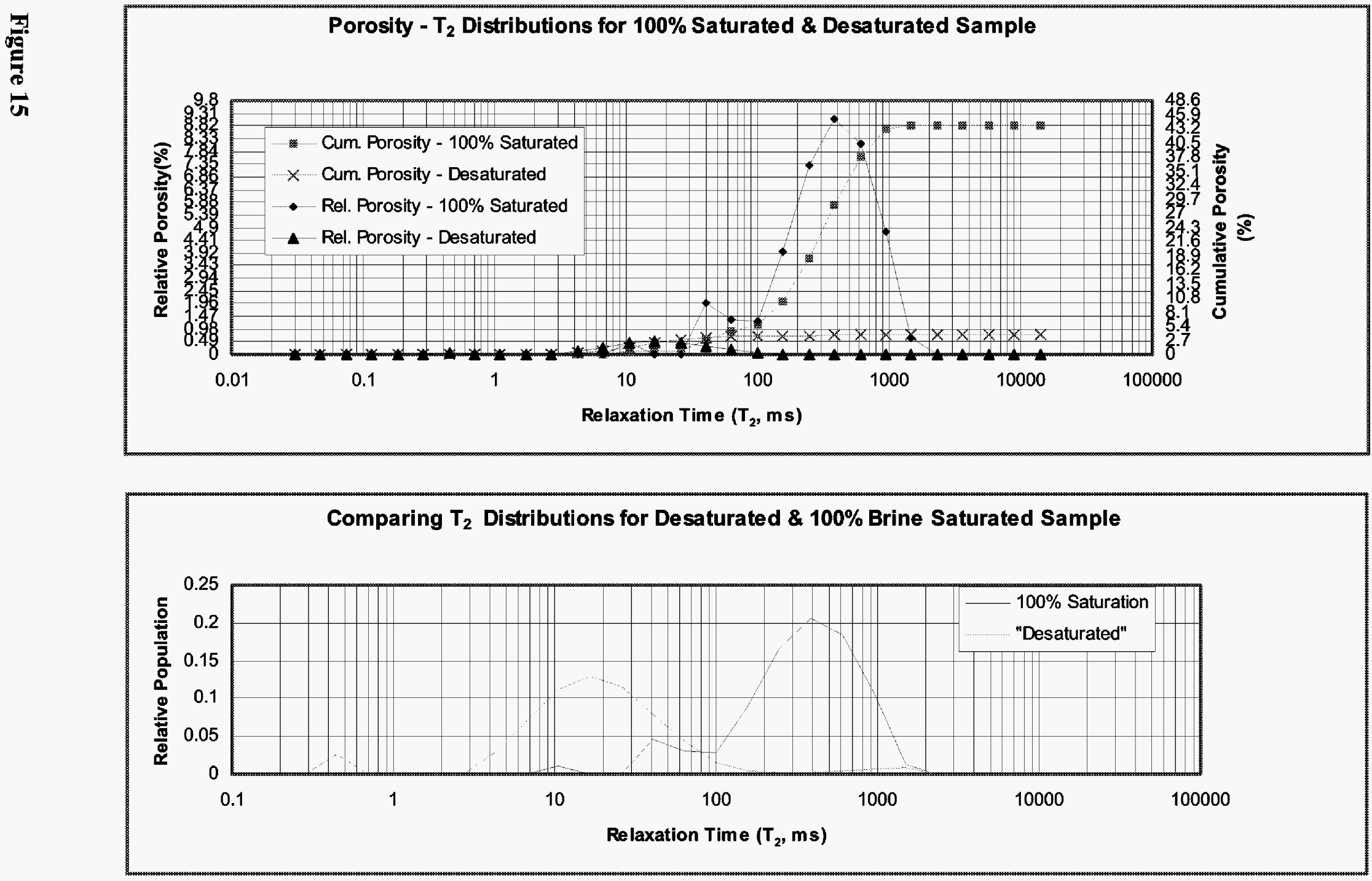

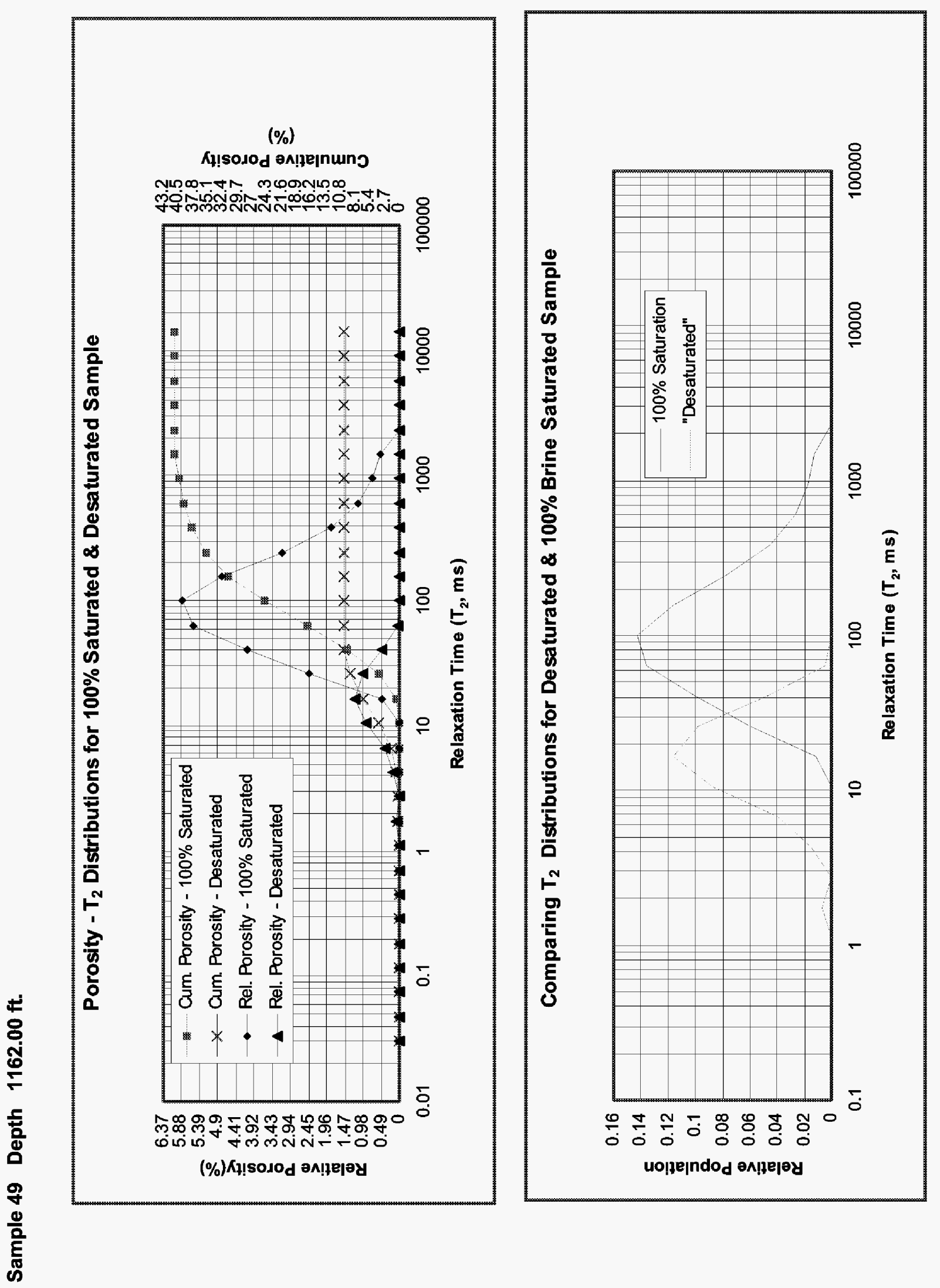

Figure 16 


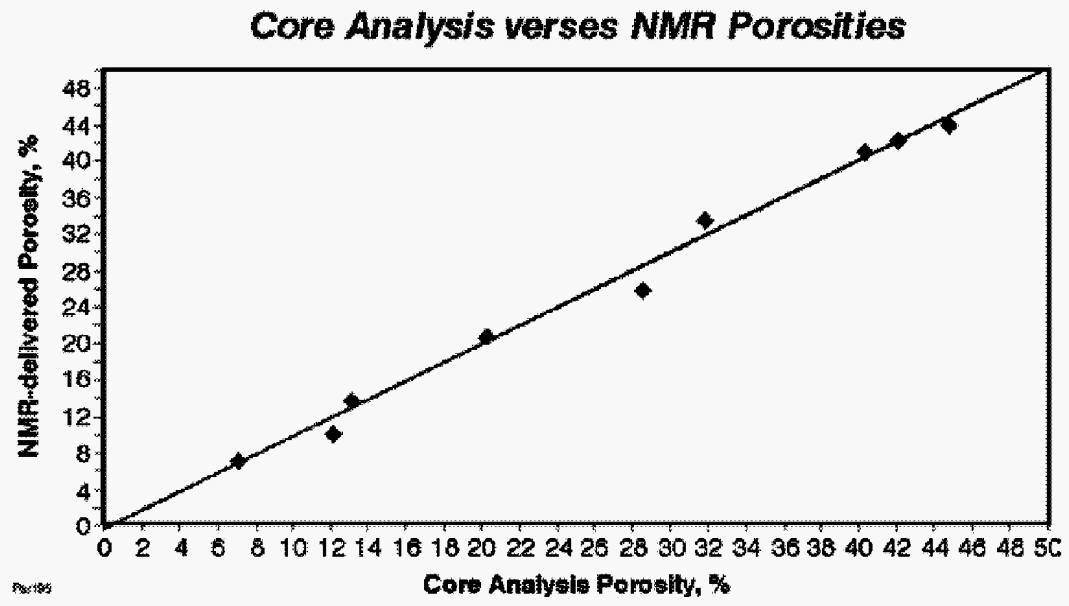

Figure 17

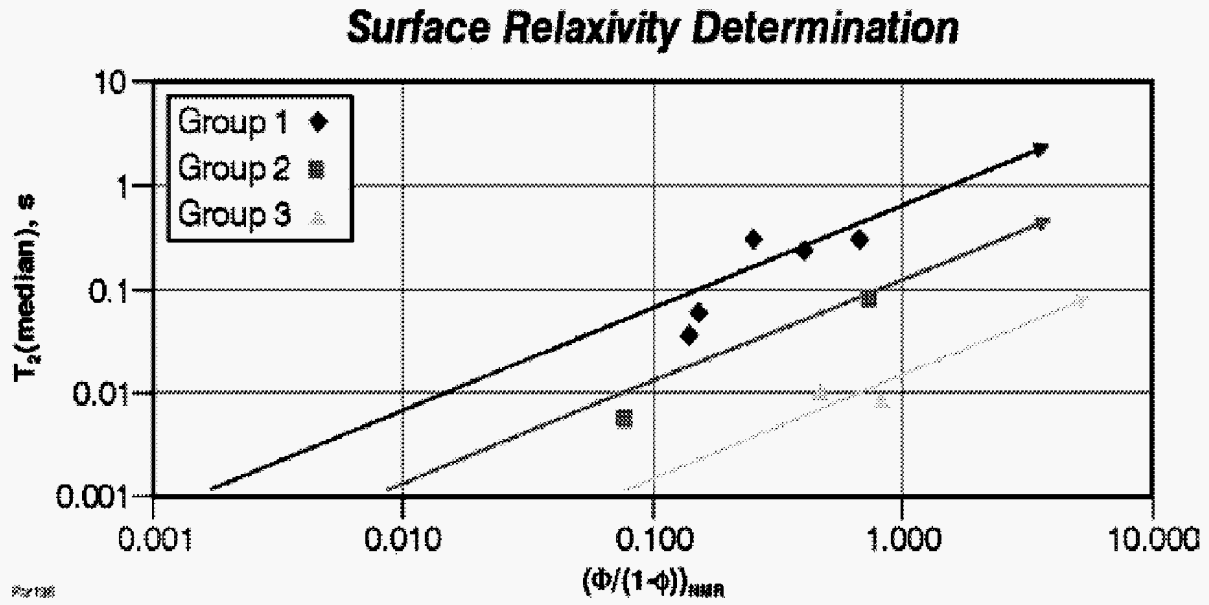

Figure 18 


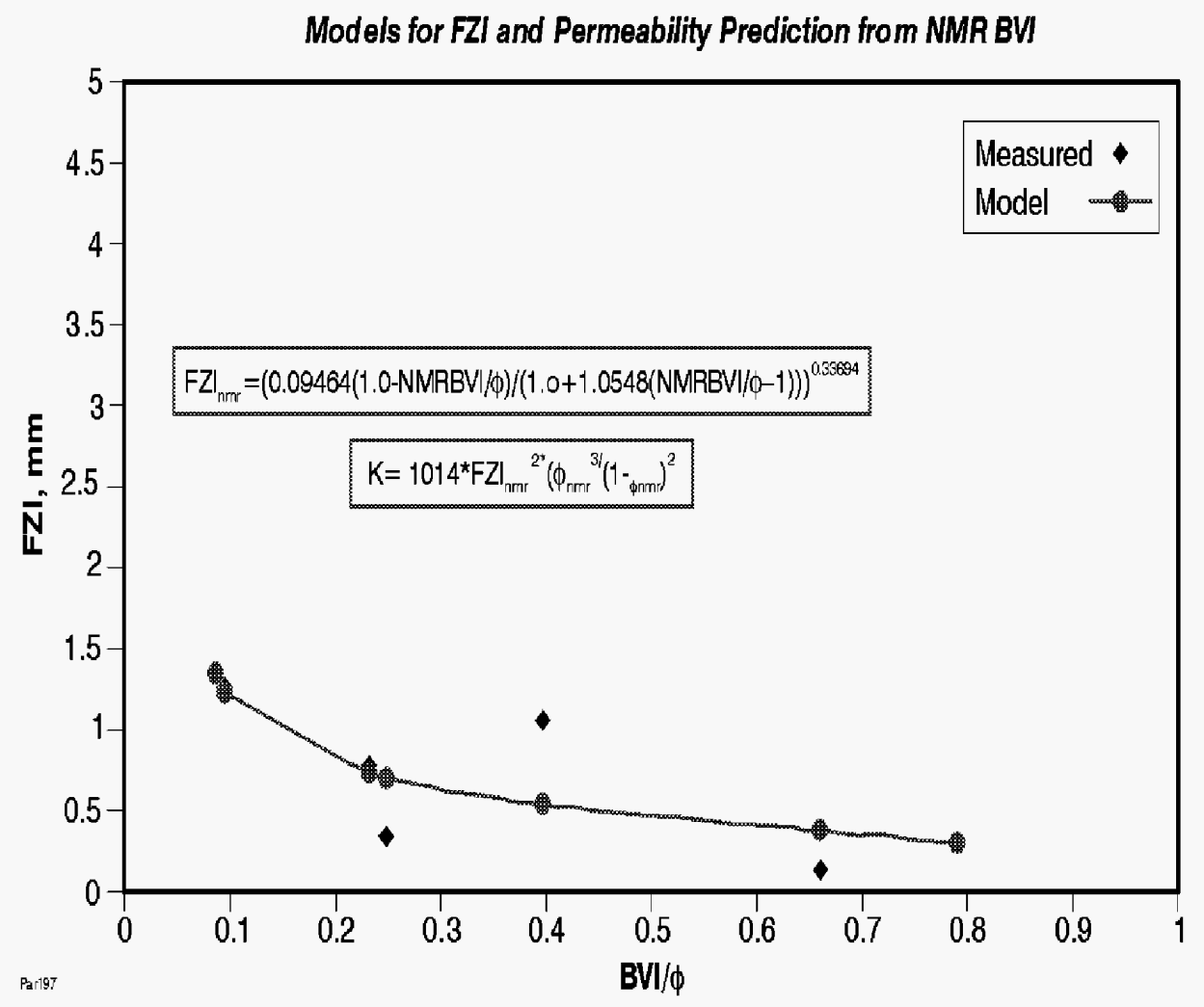

Figure 19 

